\title{
Specification for the VERA Depletion Benchmark Suite
}

\author{
Revision 0 \\ December 17, 2015
}

Kang Seog Kim
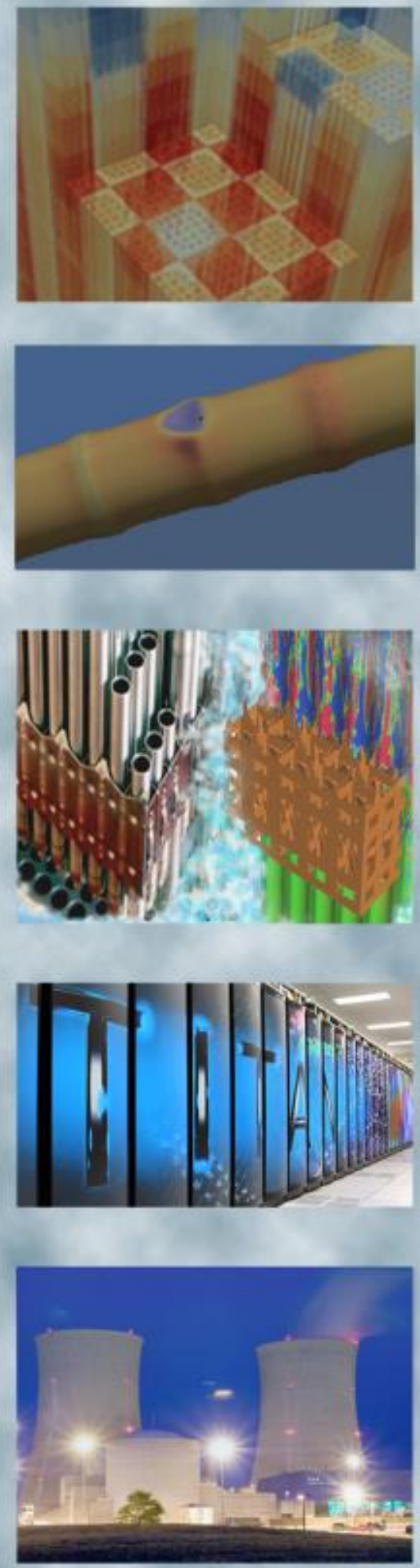


\section{REVISION LOG}

\begin{tabular}{|c|c|c|l|}
\hline Revision & Date & Affected Pages & \multicolumn{1}{c|}{ Revision Description } \\
\hline 0 & $12 / 17 / 2015$ & All & Initial version \\
\hline & & & \\
\hline & & & \\
\hline & & & \\
\hline
\end{tabular}

Document pages that are:

Export Controlled None

IP/Proprietary/NDA Controlled None

Sensitive Controlled None

Unlimited ALL PAGES

\section{Requested Distribution:}

To: N/A

Copy: N/A

\section{Reviewed by:}

Date:

Reviewer: Kevin Clarno 


\section{EXECUTIVE SUMMARY}

The CASL neutronics simulator MPACT is under development for the neutronics and T-H coupled simulation for the pressurized water reactor. MPACT includes the ORIGEN-API and internal depletion module to perform depletion calculations based upon neutron-material reaction and radioactive decay. It is a challenge to validate the depletion capability because of the insufficient measured data. One of the detoured methods to validate it is to perform a code-to-code comparison for benchmark problems. In this study a depletion benchmark suite has been developed and a detailed guideline has been provided to obtain meaningful computational outcomes which can be used in the validation of the MPACT depletion capability. 


\section{CONTENTS}

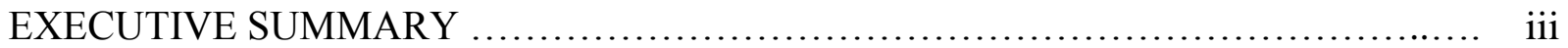

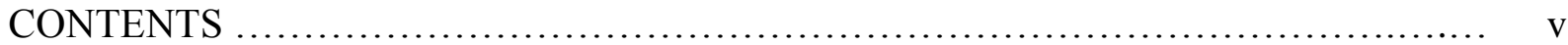

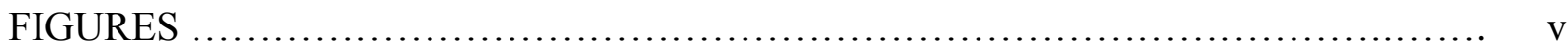

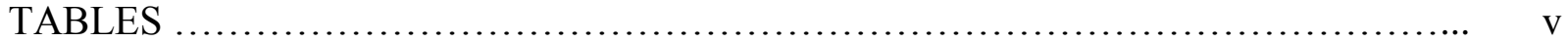

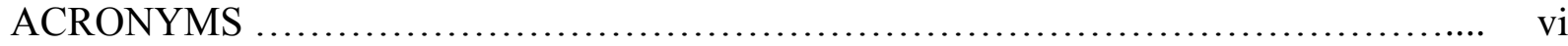

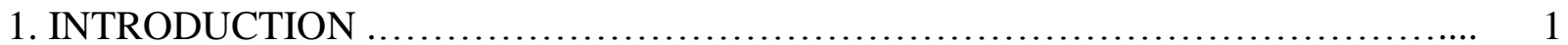

2. GEOMETRY AND MATERIAL DATA ............................................ 2

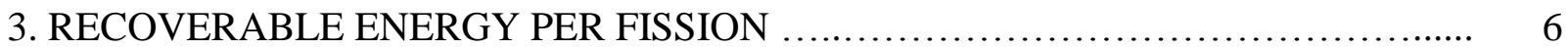

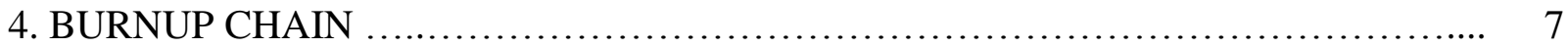

5. DEPLETION BENCHMARK PROBLEMS …................................. 8

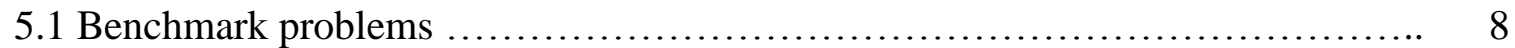

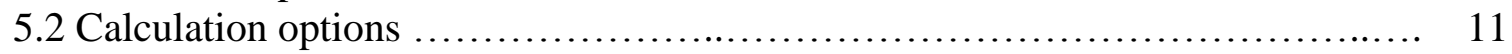

5.3 Edition of reactor physics parameters ........................................ 12

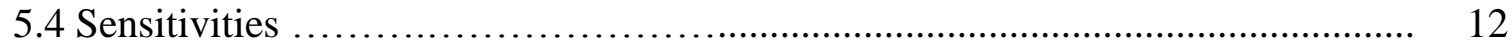

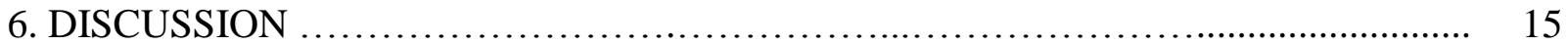

ACKNOWLEDGEMENT ......................................................... 15

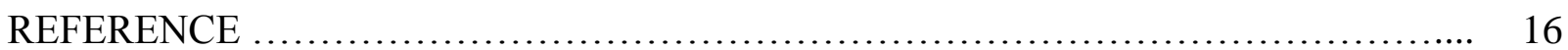

APPENDIX A. Constituent nuclides for the simplified burnup chain ................. 17

APPENDIX B. Depletion chain ................................................... 22

\section{FIGURES}

Figure 5.1 Lattice pin cell configuration

\section{TABLES}

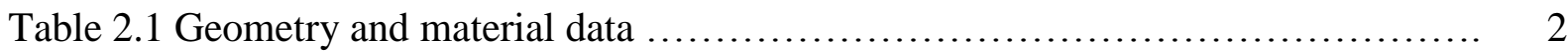

Table 2.2 Density and atomic number density for each material ......................... 4

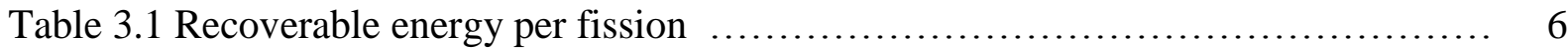

Table 5.1 Single pin and assembly depletion benchmark problems ..................... 8

Table 5.2 Multiplication factors at $0.0 \mathrm{MWD} / \mathrm{kgU}$ burnup .................................. 9

Table 5.3 Burnup points and editing options ....................................... 11

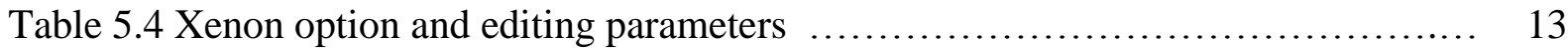

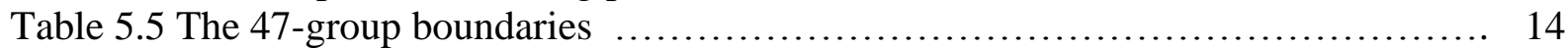

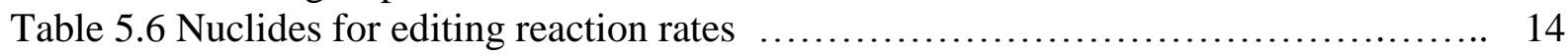

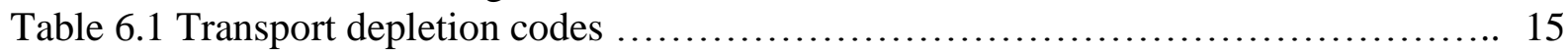




\section{ACRONYMS}

2D Two-Dimensional

CASL Consortium for Advanced Simulation of Light Water Reactors

FPY Fission Product Yield

MOC Method of Characteristics

MG Multi-group (as in cross sections)

ORNL Oak Ridge National Laboratory

PND Particle (Atomic) Number Density

PWR Pressurized water reactor

T-H Thermal Hydraulic

UM University of Michigan

VERA Virtual Environment for Reactor Applications 


\section{INTRODUCTION}

The CASL neutronics simulator MPACT is under development for the neutronics and T-H coupled simulation for the pressurized water reactor. MPACT includes the ORIGEN-API and internal depletion module to perform depletion calculations based upon neutron-material reaction and radioactive decay. It is a challenge to validate the depletion capability because of the insufficient measured data. One of the detoured methods to validate it is to perform a code-to-code comparison for depletion benchmark problems. In this study a depletion benchmark suite has been developed and a detailed guideline has been provided to obtain meaningful computational outcomes which can be used in the validation of the MPACT depletion capability.

The benchmark problems have been developed based on "The VERA Core Physics Benchmark Progression Problems" [God14]. This document provides the detailed design data and guide lines to obtain meaningful and valuable outcomes for the validation of the MPACT depletion capability.

- Geometry and composition data required in preparing user input files for the engaged simulation codes in Chapter 2,

- Recoverable energies per fission to exclude a difference of neutron flux level due to code specific recoverable energies per fission in Chapter 3,

- Burnup chains of the ORIGEN-API to figure out the differences of burnup chains with the engaged simulation programs in Chapter 4,

- Detailed description for the benchmark problems, user options and nuclear parameters to be edited and compared in Chapter 5. 


\section{GEOMETRY AND MATERIAL DATA}

The depletion benchmark problems have been developed based on the VERA progression problems [God14]. Table 2.1 provides the geometrical and material data for the benchmark problems which come from the VERA progression problems \#1 and \#2.

Table 2.2 provides density and atomic number densities for each material which should be utilized in depletion calculation to exclude a bias due to the difference in initial atomic number densities.

Table 2.1 Geometry and material data $(1 / 2)$

\begin{tabular}{|c|c|c|c|}
\hline Material & Parameter & Value & Remark \\
\hline \multirow[t]{5}{*}{ Core } & Pressure (bar) & 155.13 & $2250 \mathrm{psia}$ \\
\hline & Power Density (w/gU) & 40.00 & \\
\hline & Fuel assembly power (MW)/cm & 0.050324728 & Variable \\
\hline & Assembly Pitch $(\mathrm{cm})$ & 21.5000 & \\
\hline & Pin Pitch $(\mathrm{cm})$ & 1.2600 & \\
\hline \multirow[t]{3}{*}{ Fuel } & Pellet Radius (cm) & 0.4096 & \\
\hline & Material & $\mathrm{UO}_{2}$ & Various ${ }^{235} \mathrm{U}$ w/o \\
\hline & Density $\left(\mathrm{g} / \mathrm{cm}^{3}\right)$ & 10.2570 & \\
\hline \multirow[t]{3}{*}{ Gadolinia Rod } & Pellet Radius $(\mathrm{cm})$ & 0.4096 & \\
\hline & Material & $\mathrm{UO}_{2}+\mathrm{Gd}_{2} \mathrm{O}_{3}$ & $1.8 \mathrm{w} / \mathrm{o}^{235} \mathrm{U}, 5 \% \mathrm{Gd}_{2} \mathrm{O}_{3}$ \\
\hline & Density $\left(\mathrm{g} / \mathrm{cm}^{3}\right)$ & 10.1110 & \\
\hline \multirow[t]{4}{*}{ Clad } & Inner Radius (cm) & 0.4180 & \\
\hline & Outer Radius $(\mathrm{cm})$ & 0.4750 & \\
\hline & Material & Zircaloy-4 & \\
\hline & Density $\left(\mathrm{g} / \mathrm{cm}^{3}\right)$ & 6.5600 & \\
\hline \multirow{4}{*}{ Guide Tube } & Inner Radius (cm) & 0.5610 & \\
\hline & Outer Radius $(\mathrm{cm})$ & 0.6020 & \\
\hline & Material & Zircaloy-4 & \\
\hline & Density $\left(\mathrm{g} / \mathrm{cm}^{3}\right)$ & 6.5600 & \\
\hline \multirow{4}{*}{$\begin{array}{l}\text { Instrumentation } \\
\text { Tube }\end{array}$} & Inner Radius $(\mathrm{cm})$ & 0.5590 & \\
\hline & Outer Radius $(\mathrm{cm})$ & 0.6050 & \\
\hline & Material & Zircaloy-4 & \\
\hline & Density $\left(\mathrm{g} / \mathrm{cm}^{3}\right)$ & 6.5600 & \\
\hline \multirow{5}{*}{$\begin{array}{l}\text { Thimble inside } \\
\text { Instrumentation } \\
\text { Tube }\end{array}$} & Inner Radius of thimble $(\mathrm{cm})$ & 0.2580 & \\
\hline & Outer Radius of thimble $(\mathrm{cm})$ & 0.3820 & \\
\hline & Material & SS304 & \\
\hline & Internal material & ${ }^{4} \mathrm{He}$ & \\
\hline & Material between thimble and IT & Moderator & \\
\hline
\end{tabular}


Table 2.1 Geometrical and material data (2/2)

\begin{tabular}{|c|c|c|c|}
\hline Material & Parameter & Value & Remark \\
\hline \multirow{7}{*}{$\begin{array}{l}\text { Control Rod } \\
\left(\mathrm{AgInCd} \& \mathrm{~B}_{4} \mathrm{C}\right)\end{array}$} & AgInCd Radius (cm) & 0.3820 & \\
\hline & AgInCd Density $\left(\mathrm{g} / \mathrm{cm}^{3}\right)$ & 10.2000 & \\
\hline & $\mathrm{B}_{4} \mathrm{C}$ Radius $(\mathrm{cm})$ & 0.3730 & \\
\hline & $\mathrm{B}_{4} \mathrm{C}$ Density $\left(\mathrm{g} / \mathrm{cm}^{3}\right)$ & 1.7600 & \\
\hline & Clad Inner Radius (cm) & 0.3860 & \\
\hline & Clad Outer Radius (cm) & 0.4840 & \\
\hline & SS304 Clad Density $\left(\mathrm{g} / \mathrm{cm}^{3}\right)$ & 7.8000 & \\
\hline \multirow[t]{4}{*}{ IFBA } & Coating Thickness $(\mu \mathrm{m})$ & 10.0000 & \\
\hline & Material & $\mathrm{ZrB}_{2}$ & \\
\hline & ${ }^{10} \mathrm{~B}$ enrichment (w/o) & 50.0 & \\
\hline & Density $\left(\mathrm{g} / \mathrm{cm}^{3}\right)$ & 3.8500 & \\
\hline \multirow[t]{9}{*}{ WABA } & Material & $\mathrm{B}_{4} \mathrm{C}+\mathrm{Al}_{2} \mathrm{O}_{3}$ & \\
\hline & Density $\left(\mathrm{g} / \mathrm{cm}^{3}\right)$ & 3.6500 & \\
\hline & Inner Clad Inner Radius (cm) & 0.2860 & \\
\hline & Inner Clad Outer Radius (cm) & 0.3390 & \\
\hline & Inner/Outer Clad Material & Zircaloy-4 & \\
\hline & Poison Inner Radius (cm) & 0.3530 & \\
\hline & Poison Outer Radius $(\mathrm{cm})$ & 0.4040 & \\
\hline & Outer Clad Inner Radius (cm) & 0.4180 & \\
\hline & Outer Clad Outer Radius (cm) & 0.4840 & \\
\hline \multirow[t]{9}{*}{ Pyrex } & Material & $\mathrm{B}_{2} \mathrm{O}_{3}+\mathrm{SiO}_{2}$ & $12.5 \mathrm{w} / \mathrm{o} \mathrm{B}_{2} \mathrm{O}_{3}$ \\
\hline & Density $\left(\mathrm{g} / \mathrm{cm}^{3}\right)$ & 2.2500 & \\
\hline & Inner Clad Inner Radius (cm) & 0.2140 & \\
\hline & Inner Clad Outer Radius (cm) & 0.2310 & \\
\hline & Inner/Outer Clad Material & SS-304 & \\
\hline & Poison Inner Radius (cm) & 0.2410 & \\
\hline & Poison Outer Radius $(\mathrm{cm})$ & 0.4270 & \\
\hline & Outer Clad Inner Radius (cm) & 0.4370 & \\
\hline & Outer Clad Outer Radius (cm) & 0.4840 & \\
\hline
\end{tabular}


Table 2.2 Density and atomic number density for each material (1/2)

\begin{tabular}{|c|c|c|c|c|c|}
\hline Material & $\operatorname{Density}\left(\mathrm{g} / \mathrm{cm}^{3}\right)$ & $\begin{array}{l}\text { Isotope } \\
\text { ID }\end{array}$ & PND (/barn-cm) & Isotope ID & $\begin{array}{c}\text { PND (/barn- } \\
\mathrm{cm})\end{array}$ \\
\hline \multirow{3}{*}{ Fuel $(2.1 \%)$} & \multirow{3}{*}{10.257} & 92234 & $4.02487 \mathrm{E}-06$ & 92238 & $2.23868 \mathrm{E}-02$ \\
\hline & & 92235 & 4.86484E-04 & 8016 & $4.57590 \mathrm{E}-02$ \\
\hline & & 92236 & $2.23756 \mathrm{E}-06$ & & \\
\hline \multirow{3}{*}{ Fuel (3.1\%) } & \multirow{3}{*}{10.257} & 92234 & $6.11864 \mathrm{E}-06$ & 92238 & $2.21546 \mathrm{E}-02$ \\
\hline & & 92235 & 7.18132E-04 & 8016 & $4.57642 \mathrm{E}-02$ \\
\hline & & 92236 & $3.29861 \mathrm{E}-06$ & & \\
\hline \multirow{3}{*}{ Fuel (3.6\%) } & \multirow{3}{*}{10.257} & 92234 & $7.21203 \mathrm{E}-06$ & 92238 & 2.20384E-02 \\
\hline & & 92235 & $8.33952 \mathrm{E}-04$ & 8016 & $4.57669 \mathrm{E}-02$ \\
\hline & & 92236 & $3.82913 \mathrm{E}-06$ & & \\
\hline \multirow{3}{*}{ Fuel $(4.6 \%)$} & \multirow{3}{*}{10.257} & 92234 & $9.39876 \mathrm{E}-06$ & 92238 & $2.18062 \mathrm{E}-02$ \\
\hline & & 92235 & $1.06559 \mathrm{E}-03$ & 8016 & $4.57721 \mathrm{E}-02$ \\
\hline & & 92236 & 4.89014E-06 & & \\
\hline Gap & $1.79 \mathrm{E}-04$ & 2004 & $2.68714 \mathrm{E}-05$ & & \\
\hline \multirow{15}{*}{ Cladding (Zirc-4) } & \multirow{15}{*}{6.56} & 40090 & $2.18865 \mathrm{E}-02$ & 26054 & $8.68307 \mathrm{E}-06$ \\
\hline & & 40091 & $4.77292 \mathrm{E}-03$ & 26056 & $1.36306 \mathrm{E}-04$ \\
\hline & & 40092 & 7.29551E-03 & 26057 & $3.14789 \mathrm{E}-06$ \\
\hline & & 40094 & $7.39335 \mathrm{E}-03$ & 26058 & $4.18926 \mathrm{E}-07$ \\
\hline & & 40096 & $1.19110 \mathrm{E}-03$ & 24050 & $3.30121 \mathrm{E}-06$ \\
\hline & & 50112 & $4.68066 \mathrm{E}-06$ & 24052 & $6.36606 \mathrm{E}-05$ \\
\hline & & 50114 & $3.18478 \mathrm{E}-06$ & 24053 & $7.21860 \mathrm{E}-06$ \\
\hline & & 50115 & $1.64064 \mathrm{E}-06$ & 24054 & $1.79686 \mathrm{E}-06$ \\
\hline & & 50116 & $7.01616 \mathrm{E}-05$ & 72174 & $3.54138 \mathrm{E}-09$ \\
\hline & & 50117 & 3.70592E-05 & 72176 & $1.16423 \mathrm{E}-07$ \\
\hline & & 50118 & $1.16872 \mathrm{E}-04$ & 72177 & $4.11686 \mathrm{E}-07$ \\
\hline & & 50119 & $4.14504 \mathrm{E}-05$ & 72178 & $6.03806 \mathrm{E}-07$ \\
\hline & & 50120 & $1.57212 \mathrm{E}-04$ & 72179 & $3.01460 \mathrm{E}-07$ \\
\hline & & 50122 & $2.23417 \mathrm{E}-05$ & 72180 & $7.76449 \mathrm{E}-07$ \\
\hline & & 50124 & $2.79392 \mathrm{E}-05$ & & \\
\hline \multirow{2}{*}{$\begin{array}{c}\text { Moderator } 565 \mathrm{~K} \\
1300 \mathrm{ppm}\end{array}$} & \multirow{2}{*}{0.743} & 8016 & $2.48112 \mathrm{E}-02$ & 5010 & $1.07070 \mathrm{E}-05$ \\
\hline & & 1001 & 4.96224E-02 & 5011 & 4.30971E-05 \\
\hline \multirow{2}{*}{$\begin{array}{c}\text { Moderator } 600 \mathrm{~K} \\
1300 \mathrm{ppm}\end{array}$} & \multirow{2}{*}{0.700} & 8016 & $2.33753 \mathrm{E}-02$ & 5010 & $1.00874 \mathrm{E}-05$ \\
\hline & & 1001 & $4.67505 \mathrm{E}-02$ & 5011 & $4.06030 \mathrm{E}-05$ \\
\hline \multirow{4}{*}{ IFBA } & \multirow{4}{*}{3.85} & 5010 & $2.16410 \mathrm{E}-02$ & 40092 & $3.54348 \mathrm{E}-03$ \\
\hline & & 5011 & $1.96824 \mathrm{E}-02$ & 40094 & $3.59100 \mathrm{E}-03$ \\
\hline & & 40090 & $1.06304 \mathrm{E}-02$ & 40096 & $5.78528 \mathrm{E}-04$ \\
\hline & & 40091 & $2.31824 \mathrm{E}-03$ & & \\
\hline \multirow{3}{*}{ Pyrex } & \multirow{3}{*}{2.25} & 5010 & $9.63266 \mathrm{E}-04$ & 14028 & $1.81980 \mathrm{E}-02$ \\
\hline & & 5011 & $3.90172 \mathrm{E}-03$ & 14029 & $9.24474 \mathrm{E}-04$ \\
\hline & & 8016 & $4.67761 \mathrm{E}-02$ & 14030 & $6.10133 \mathrm{E}-04$ \\
\hline \multirow{6}{*}{$\begin{array}{c}\text { Gadolinia } \\
5 \% \mathrm{Gd}_{2} \mathrm{O}_{3} \\
95 \% \mathrm{UO}_{2} \\
1.8 \%{ }^{235} \mathrm{U} \text { Fuel }\end{array}$} & \multirow{6}{*}{10.111} & 92234 & $3.18096 \mathrm{E}-06$ & 64155 & $2.48606 \mathrm{E}-04$ \\
\hline & & 92235 & $3.90500 \mathrm{E}-04$ & 64156 & 3.43849E-04 \\
\hline & & 92236 & $1.79300 \mathrm{E}-06$ & 64157 & $2.62884 \mathrm{E}-04$ \\
\hline & & 92238 & $2.10299 \mathrm{E}-02$ & 64158 & $4.17255 \mathrm{E}-04$ \\
\hline & & 64152 & $3.35960 \mathrm{E}-06$ & 64160 & $3.67198 \mathrm{E}-04$ \\
\hline & & 64154 & $3.66190 \mathrm{E}-05$ & 8016 & $4.53705 \mathrm{E}-02$ \\
\hline
\end{tabular}


Table 2.2 Density and atomic number density for each material (2/2)

\begin{tabular}{|c|c|c|c|c|c|}
\hline Material & Density $\left(\mathrm{g} / \mathrm{cm}^{3}\right)$ & Isotope ID & PND (/barn-cm) & Isotope ID & PND (/barn-cm) \\
\hline \multirow{10}{*}{ SS304 } & \multirow{10}{*}{7.8} & 6000 & $3.20895 \mathrm{E}-04$ & 26054 & $3.44776 \mathrm{E}-03$ \\
\hline & & 14028 & $1.58197 \mathrm{E}-03$ & 26056 & $5.41225 \mathrm{E}-02$ \\
\hline & & 14029 & $8.03653 \mathrm{E}-05$ & 26057 & $1.24992 \mathrm{E}-03$ \\
\hline & & 14030 & $5.30394 \mathrm{E}-05$ & 26058 & $1.66342 \mathrm{E}-04$ \\
\hline & & 15031 & $6.99938 \mathrm{E}-05$ & 28058 & $5.30854 \mathrm{E}-03$ \\
\hline & & 24050 & 7.64915E-04 & 28060 & $2.04484 \mathrm{E}-03$ \\
\hline & & 24052 & $1.47506 \mathrm{E}-02$ & 28061 & 8.88879E-05 \\
\hline & & 24053 & $1.67260 \mathrm{E}-03$ & 28062 & $2.83413 \mathrm{E}-04$ \\
\hline & & 24054 & $4.16346 \mathrm{E}-04$ & 28064 & $7.21770 \mathrm{E}-05$ \\
\hline & & 25055 & $1.75387 \mathrm{E}-03$ & & \\
\hline \multirow{6}{*}{$\mathrm{AgInCd}$} & \multirow{6}{*}{10.2} & 47107 & $2.36159 \mathrm{E}-02$ & 48112 & $6.59276 \mathrm{E}-04$ \\
\hline & & 47109 & 2.19403E-02 & 48113 & 3.33873E-04 \\
\hline & & 48106 & $3.41523 \mathrm{E}-05$ & 48114 & $7.84957 \mathrm{E}-04$ \\
\hline & & 48108 & $2.43165 \mathrm{E}-05$ & 48116 & $2.04641 \mathrm{E}-04$ \\
\hline & & 48110 & $3.41250 \mathrm{E}-04$ & 49113 & $3.44262 \mathrm{E}-04$ \\
\hline & & 48111 & $3.49720 \mathrm{E}-04$ & 49115 & $7.68050 \mathrm{E}-03$ \\
\hline \multirow{2}{*}{$\mathrm{B}_{4} \mathrm{C}$} & \multirow{2}{*}{1.76} & 5010 & $1.52689 \mathrm{E}-02$ & 6000 & 1.91820E-02 \\
\hline & & 5011 & 6.14591E-02 & & \\
\hline \multirow{3}{*}{$\begin{array}{c}\text { WABA } \\
\mathrm{B}_{4} \mathrm{C}-\mathrm{Al}_{2} \mathrm{O}_{3}\end{array}$} & \multirow{3}{*}{3.65} & 5010 & $2.98553 \mathrm{E}-03$ & 8016 & 5.85563E-02 \\
\hline & & 5011 & $1.21192 \mathrm{E}-02$ & 13027 & $3.90223 \mathrm{E}-02$ \\
\hline & & 6000 & $3.77001 \mathrm{E}-03$ & & \\
\hline
\end{tabular}




\section{RECOVERABLE ENERGY PER FISSION}

The neutron flux level would impact on predicting isotopic inventories at burnup calculation which is estimated by using the code specific recoverable energies per fission. Therefore, it is recommended to utilize the common recoverable energy per fission provided in Table 3.1.

Table 3.1 Recoverable energy per fission

\begin{tabular}{|c|c|c|c|c|c|c|}
\hline \multirow{2}{*}{ No } & \multirow{2}{*}{ ID } & \multirow{2}{*}{ Nuclide } & \multicolumn{2}{|c|}{ ENDF/B-7.0 } & \multicolumn{2}{|c|}{ ENDF/B-7.1 } \\
\hline & & & Kappa(w-s) & Kappa0(w-s) & Kappa(w-s) & Kappa0(w-s) \\
\hline 1 & 90230 & ${ }^{230} \mathrm{Th}$ & $3.24352 \mathrm{E}-11$ & $3.05372 \mathrm{E}-11$ & $3.22841 \mathrm{E}-11$ & $2.89031 \mathrm{E}-11$ \\
\hline 2 & 90232 & ${ }^{232} \mathrm{Th}$ & $3.22345 \mathrm{E}-11$ & $3.01965 \mathrm{E}-11$ & $3.22345 \mathrm{E}-11$ & $3.01965 \mathrm{E}-11$ \\
\hline 3 & 91231 & ${ }^{231} \mathrm{~Pa}$ & $3.38655 \mathrm{E}-11$ & $3.06082 \mathrm{E}-11$ & $3.38655 \mathrm{E}-11$ & $2.97356 \mathrm{E}-11$ \\
\hline 4 & 91233 & ${ }^{233} \mathrm{~Pa}$ & $3.27020 \mathrm{E}-11$ & $3.06082 \mathrm{E}-11$ & $3.26046 \mathrm{E}-11$ & $2.97452 \mathrm{E}-11$ \\
\hline 5 & 92232 & ${ }^{232} \mathrm{U}$ & $3.40754 \mathrm{E}-11$ & $3.06082 \mathrm{E}-11$ & $3.40754 \mathrm{E}-11$ & $2.95739 \mathrm{E}-11$ \\
\hline 6 & 92233 & ${ }^{233} \mathrm{U}$ & $3.20712 \mathrm{E}-11$ & $3.06082 \mathrm{E}-11$ & $3.20712 \mathrm{E}-11$ & $3.06082 \mathrm{E}-11$ \\
\hline 7 & 92234 & ${ }^{234} \mathrm{U}$ & $3.26494 \mathrm{E}-11$ & $3.07364 \mathrm{E}-11$ & 3.26494E-11 & $3.07364 \mathrm{E}-11$ \\
\hline 8 & 92235 & ${ }^{235} \mathrm{U}$ & $3.24187 \mathrm{E}-11$ & $3.09997 \mathrm{E}-11$ & $3.24187 \mathrm{E}-11$ & $3.09872 \mathrm{E}-11$ \\
\hline 9 & 92236 & ${ }^{236} \mathrm{U}$ & $3.30019 \mathrm{E}-11$ & $3.11610 \mathrm{E}-11$ & $3.30019 \mathrm{E}-11$ & $3.11610 \mathrm{E}-11$ \\
\hline 10 & 92237 & ${ }^{237} \mathrm{U}$ & $3.36652 \mathrm{E}-11$ & $3.14446 \mathrm{E}-11$ & $3.36652 \mathrm{E}-11$ & $3.00925 \mathrm{E}-11$ \\
\hline 11 & 92238 & ${ }^{238} \mathrm{U}$ & $3.40626 \mathrm{E}-11$ & $3.17285 \mathrm{E}-11$ & $3.40626 \mathrm{E}-11$ & $3.16891 \mathrm{E}-11$ \\
\hline 12 & 92240 & ${ }^{240} \mathrm{U}$ & $3.33592 \mathrm{E}-11$ & $3.17330 \mathrm{E}-11$ & 3.33592E-11 & $3.17330 \mathrm{E}-11$ \\
\hline 13 & 93237 & ${ }^{237} \mathrm{~Np}$ & $3.37405 \mathrm{E}-11$ & 3.14622E-11 & $3.37405 \mathrm{E}-11$ & 3.14622E-11 \\
\hline 14 & 93238 & ${ }^{238} \mathrm{~Np}$ & $3.37181 \mathrm{E}-11$ & $3.18579 \mathrm{E}-11$ & $3.37181 \mathrm{E}-11$ & $3.19722 \mathrm{E}-11$ \\
\hline 15 & 93239 & ${ }^{239} \mathrm{~Np}$ & $3.42660 \mathrm{E}-11$ & $3.18579 \mathrm{E}-11$ & $3.42660 \mathrm{E}-11$ & $3.04126 \mathrm{E}-11$ \\
\hline 16 & 94236 & ${ }^{236} \mathrm{Pu}$ & $3.29971 \mathrm{E}-11$ & $3.11610 \mathrm{E}-11$ & 3.34433E-11 & 3.19691E-11 \\
\hline 17 & 94237 & ${ }^{237} \mathrm{Pu}$ & $3.30131 \mathrm{E}-11$ & $3.13869 \mathrm{E}-11$ & $3.38885 \mathrm{E}-11$ & $3.22623 \mathrm{E}-11$ \\
\hline 18 & 94238 & ${ }^{238} \mathrm{Pu}$ & $3.36748 \mathrm{E}-11$ & $3.16240 \mathrm{E}-11$ & $3.41258 \mathrm{E}-11$ & $3.21010 \mathrm{E}-11$ \\
\hline 19 & 94239 & ${ }^{239} \mathrm{Pu}$ & 3.43312E-11 & $3.18586 \mathrm{E}-11$ & 3.43312E-11 & 3.18679E-11 \\
\hline 20 & 94240 & ${ }^{240} \mathrm{Pu}$ & $3.43157 \mathrm{E}-11$ & $3.19589 \mathrm{E}-11$ & $3.43157 \mathrm{E}-11$ & $3.19589 \mathrm{E}-11$ \\
\hline 21 & 94241 & ${ }^{241} \mathrm{Pu}$ & $3.47426 \mathrm{E}-11$ & $3.23611 \mathrm{E}-11$ & $3.47426 \mathrm{E}-11$ & $3.23611 \mathrm{E}-11$ \\
\hline 22 & 94242 & ${ }^{242} \mathrm{Pu}$ & 3.47643E-11 & $3.22969 \mathrm{E}-11$ & 3.49302E-11 & $3.24889 \mathrm{E}-11$ \\
\hline 23 & 94244 & ${ }^{244} \mathrm{Pu}$ & $3.41266 \mathrm{E}-11$ & $3.25004 \mathrm{E}-11$ & $3.35567 \mathrm{E}-11$ & 3.19305E-11 \\
\hline 24 & 95241 & ${ }^{241} \mathrm{Am}$ & $3.48348 \mathrm{E}-11$ & $3.23578 \mathrm{E}-11$ & $3.48348 \mathrm{E}-11$ & $3.23578 \mathrm{E}-11$ \\
\hline 25 & 95242 & ${ }^{242} \mathrm{Am}$ & $3.45085 \mathrm{E}-11$ & $3.22969 \mathrm{E}-11$ & $3.51714 \mathrm{E}-11$ & $3.29599 \mathrm{E}-11$ \\
\hline 26 & 95243 & ${ }^{243} \mathrm{Am}$ & $3.56147 \mathrm{E}-11$ & $3.26238 \mathrm{E}-11$ & $3.56147 \mathrm{E}-11$ & $3.26238 \mathrm{E}-11$ \\
\hline 27 & $95242 \mathrm{~m}$ & ${ }^{242 \mathrm{~m}} \mathrm{Am}$ & $3.56147 \mathrm{E}-11$ & $3.26238 \mathrm{E}-11$ & $3.56147 \mathrm{E}-11$ & $3.26238 \mathrm{E}-11$ \\
\hline 28 & 96241 & ${ }^{241} \mathrm{Cm}$ & $3.40465 \mathrm{E}-11$ & 3.24203E-11 & $3.51880 \mathrm{E}-11$ & 3.35618E-11 \\
\hline 29 & 96242 & ${ }^{242} \mathrm{Cm}$ & $3.54821 \mathrm{E}-11$ & $3.24524 \mathrm{E}-11$ & $3.54821 \mathrm{E}-11$ & $3.25983 \mathrm{E}-11$ \\
\hline 30 & 96243 & ${ }^{243} \mathrm{Cm}$ & $3.50159 \mathrm{E}-11$ & $3.24764 \mathrm{E}-11$ & $3.50159 \mathrm{E}-11$ & $3.26885 \mathrm{E}-11$ \\
\hline 31 & 96244 & ${ }^{244} \mathrm{Cm}$ & $3.54308 \mathrm{E}-11$ & 3.25004E-11 & $3.54308 \mathrm{E}-11$ & 3.33858E-11 \\
\hline 32 & 96245 & ${ }^{245} \mathrm{Cm}$ & $3.45048 \mathrm{E}-11$ & 3.27359E-11 & $3.45060 \mathrm{E}-11$ & 3.28798E-11 \\
\hline 33 & 96246 & ${ }^{246} \mathrm{Cm}$ & $3.51248 \mathrm{E}-11$ & 3.29731E-11 & $3.54721 \mathrm{E}-11$ & 3.37309E-11 \\
\hline 34 & 96247 & ${ }^{247} \mathrm{Cm}$ & $3.48348 \mathrm{E}-11$ & $3.32086 \mathrm{E}-11$ & $3.51698 \mathrm{E}-11$ & $3.35436 \mathrm{E}-11$ \\
\hline 35 & 96248 & ${ }^{248} \mathrm{Cm}$ & 3.50703E-11 & 3.34441E-11 & 3.55936E-11 & 3.39674E-11 \\
\hline 36 & 97249 & ${ }^{249} \mathrm{Bk}$ & 3.50703E-11 & 3.34441E-11 & 3.60559E-11 & 3.44297E-11 \\
\hline 37 & 98249 & ${ }^{249} \mathrm{Cf}$ & $3.50703 \mathrm{E}-11$ & $3.34441 \mathrm{E}-11$ & $3.55494 \mathrm{E}-11$ & $3.39232 \mathrm{E}-11$ \\
\hline 38 & 98250 & ${ }^{250} \mathrm{Cf}$ & 3.50703E-11 & 3.34441E-11 & 3.68134E-11 & 3.51872E-11 \\
\hline 39 & 98251 & ${ }^{251} \mathrm{Cf}$ & $3.50703 \mathrm{E}-11$ & $3.34441 \mathrm{E}-11$ & $3.58147 \mathrm{E}-11$ & $3.41885 \mathrm{E}-11$ \\
\hline 40 & 98252 & ${ }^{252} \mathrm{Cf}$ & 3.50703E-11 & 3.34441E-11 & 3.68984E-11 & $3.52721 \mathrm{E}-11$ \\
\hline
\end{tabular}




\section{BURNUP CHAIN}

Typically deterministic/Monte Carlo programs include their own burnup chains and libraries. The SCALE-ORIGEN and CASL-ORIEGN include two different burnup chains which are full and simplified ones. The simplified burnup chain has been developed to include 255 nuclides and to have very good agreement with the full burnup chain especially for the multiplication factor. Since the latest SCALE-ORIGEN burnup chains and libraries reflect the most up-to-date data and methodology, it is recommended to utilize the ORIGEN-API burnup chains and libraries for benchmark calculations. The SCALE-ORIGEN libraries will be provided upon request for which the SCALE license should be acquired at first. If the program is not ready yet to handle the SCALE-ORIGEN libraries, it is required to include its own burnup chain and libraries in the report.

Appendices A and B provide constituent 255 nuclides for the simplified burnup chain and each column includes the following indicators.

- CAT: Category of nuclides

1 : Activation nuclides

2 : Heavy metal nuclides

3 : Fission product nuclides

- ID: Numeric nuclide ID in ORIGEN = ZZZAAAI

ZZZ : Atomic number

AAA : Atomic mass number

I : Stable or meta-stable (0: stable; 1 : meta)

- IFPY: Indicator for fission product yield

0 : Non FPY

1 : Direct FPY

2: Cumulative FPY

3 : Special treatment with weight fractions $\left(\mathrm{W}_{\mathrm{i}}\right)$ 


\section{DEPLETION BENCHMARK PROBLEMS}

\subsection{Benchmark problems}

The depletion benchmark suite includes 9 single pin and 16 fuel assembly problems with various fuel temperatures, ${ }^{235} \mathrm{U}$ enrichments, control rods and burnable poisons as shown in Table 5.1. The pin configurations of fuel rods, guide/instrument tubes and burnable poisons are shown in Figure 5.1. The geometrical and material data for the benchmark problems are provided in Chapter 2. In order to verify the input data prepared by participant Table 5.2 provides the multiplication factors obtained by KENO-CE with ENDF/B-7.0 at $0.0 \mathrm{MWD} / \mathrm{kgU}$.

Table 5.1 Single pin and assembly depletion benchmark problems

\begin{tabular}{|c|c|c|c|c|c|c|c|}
\hline \multirow{2}{*}{ Problem } & \multirow{2}{*}{ Description } & \multicolumn{3}{|c|}{ Temperature $(\mathrm{K})$} & \multirow{2}{*}{$\begin{array}{c}\text { Moderator } \\
\text { Density }\end{array}$} & \multirow{2}{*}{${ }^{235} \mathrm{U}$ w/o } & \multirow{2}{*}{$\begin{array}{c}\text { Power density } \\
(\mathrm{w} / \mathrm{gU})\end{array}$} \\
\hline & & Moderator & Clad & Fuel & & & \\
\hline $1 \mathrm{~A}$ & Pin $3.1 \mathrm{w} / \mathrm{o} \mathrm{T}_{\mathrm{F}}=565 \mathrm{~K}$ & 565 & 565 & 565 & 0.743 & 3.1 & 40.0 \\
\hline 1B & Pin $3.1 \mathrm{w} / \mathrm{o} \mathrm{T}_{\mathrm{F}}=600 \mathrm{~K}$ & 600 & 600 & 600 & 0.700 & 3.1 & 40.0 \\
\hline $1 \mathrm{C}$ & Pin $3.1 \mathrm{w} / \mathrm{o} \mathrm{T}_{\mathrm{F}}=900 \mathrm{~K}$ & 600 & 600 & 900 & 0.700 & 3.1 & 40.0 \\
\hline 1D & Pin $3.1 \mathrm{w} / \mathrm{o} \mathrm{T}_{\mathrm{F}}=1200 \mathrm{~K}$ & 600 & 600 & 1200 & 0.700 & 3.1 & 40.0 \\
\hline $1 \mathrm{E}$ & Pin IFBA $3.1 \mathrm{w} / \mathrm{o} \mathrm{T}_{\mathrm{F}}=600 \mathrm{~K}$ & 600 & 600 & 900 & 0.700 & 3.1 & 40.0 \\
\hline $1 \mathrm{~F}$ & Pin $2.1 \mathrm{w} / \mathrm{o} \mathrm{T}_{\mathrm{F}}=900 \mathrm{~K}$ & 600 & 600 & 900 & 0.700 & 2.1 & 40.0 \\
\hline $1 \mathrm{G}$ & Pin $3.6 \mathrm{w} / \mathrm{o} \mathrm{T}_{\mathrm{F}}=900 \mathrm{~K}$ & 600 & 600 & 900 & 0.700 & 3.6 & 40.0 \\
\hline $1 \mathrm{H}$ & Pin $4.6 \mathrm{w} / \mathrm{o} \mathrm{T}_{\mathrm{F}}=900 \mathrm{~K}$ & 600 & 600 & 900 & 0.700 & 4.6 & 40.0 \\
\hline $1 \mathrm{I}$ & Pin Gadolinia rod $\left(5 \% \mathrm{Gd}_{2} \mathrm{O}_{3}\right)$ & 600 & 600 & 900 & 0.700 & 1.8 & 40.0 \\
\hline $1 \mathrm{~J}$ & Pin $3.1 \mathrm{w} / \mathrm{o} \mathrm{T}_{\mathrm{F}}=600 / 900 / 1200 \mathrm{~K}$ & 600 & 600 & $600 / 900 / 1200$ & 0.700 & 3.1 & 40.0 \\
\hline $2 \mathrm{~A}$ & FA No Poisons $T_{\mathrm{F}}=565 \mathrm{~K}$ & 565 & 565 & 565 & 0.743 & 3.1 & 40.0 \\
\hline $2 \mathrm{~B}$ & FA No Poisons $T_{\mathrm{F}}=600 \mathrm{~K}$ & 600 & 600 & 600 & 0.700 & 3.1 & 40.0 \\
\hline $2 \mathrm{C}$ & FA No Poisons $T_{F}=900 K$ & 600 & 600 & 900 & 0.700 & 3.1 & 40.0 \\
\hline $2 \mathrm{D}$ & FA No Poisons $T_{F}=1200 K$ & 600 & 600 & 1200 & 0.700 & 3.1 & 40.0 \\
\hline $2 \mathrm{E}$ & FA 12 Pyrex & 600 & 600 & 900 & 0.700 & 3.1 & 40.0 \\
\hline $2 \mathrm{~F}$ & FA 24 Pyrex & 600 & 600 & 900 & 0.700 & 3.1 & 40.0 \\
\hline $2 \mathrm{G}$ & FA 24 AIC & 600 & 600 & 900 & 0.700 & 3.1 & 40.0 \\
\hline $2 \mathrm{H}$ & FA 24 B4C & 600 & 600 & 900 & 0.700 & 3.1 & 40.0 \\
\hline $2 \mathrm{I}$ & FA Instrument Thimble & 600 & 600 & 900 & 0.700 & 3.1 & 40.0 \\
\hline $2 \mathrm{~J}$ & FA Instrument + 24 Pyrex & 600 & 600 & 900 & 0.700 & 3.1 & 40.0 \\
\hline $2 \mathrm{~K}$ & FA Zoned + 24 Pyrex & 600 & 600 & 900 & 0.700 & $3.1 / 3.6$ & 40.0 \\
\hline $2 \mathrm{~L}$ & FA 80 IFBA & 600 & 600 & 900 & 0.700 & 3.1 & 40.0 \\
\hline $2 \mathrm{M}$ & FA 128 IFBA & 600 & 600 & 900 & 0.700 & 3.1 & 40.0 \\
\hline $2 \mathrm{~N}$ & FA 104 IFBA + 20 WABA & 600 & 600 & 900 & 0.700 & 3.1 & 40.0 \\
\hline 20 & FA 12 Gadolinia & 600 & 600 & 900 & 0.700 & $3.1 / 1.8$ & 40.0 \\
\hline $2 \mathrm{P}$ & FA 24 Gadolinia & 600 & 600 & 900 & 0.700 & $3.1 / 1.8$ & 40.0 \\
\hline
\end{tabular}


Table 5.2 Multiplication factors at 0.0 MWD/kgU burnup

\begin{tabular}{|c|c|c|c|c|c|}
\hline \multirow{2}{*}{ Problem } & \multirow{2}{*}{ Description } & \multicolumn{3}{|c|}{ Temperature $(\mathrm{K})$} & \multirow{2}{*}{ KENO-CE keff } \\
\hline & & Moderator & Clad & Fuel & \\
\hline $1 \mathrm{~A}$ & Pin $3.1 \mathrm{w} / \mathrm{o} \mathrm{T}_{\mathrm{F}}=565 \mathrm{~K}$ & 565 & 565 & 565 & $1.18704 \pm 0.00005$ \\
\hline 1B & Pin $3.1 \mathrm{w} / \mathrm{o} \mathrm{T}_{\mathrm{F}}=600 \mathrm{~K}$ & 600 & 600 & 600 & $1.18428 \pm 0.00017$ \\
\hline $1 \mathrm{C}$ & Pin $3.1 \mathrm{w} / \mathrm{o} \mathrm{T}_{\mathrm{F}}=900 \mathrm{~K}$ & 600 & 600 & 900 & $1.17393 \pm 0.00016$ \\
\hline $1 \mathrm{D}$ & Pin $3.1 \mathrm{w} / \mathrm{o} \mathrm{T}_{\mathrm{F}}=1200 \mathrm{~K}$ & 600 & 600 & 1200 & $1.16516 \pm 0.00013$ \\
\hline $1 \mathrm{E}$ & Pin IFBA $3.1 \mathrm{w} / \mathrm{o} \mathrm{T}_{\mathrm{F}}=600 \mathrm{~K}$ & 600 & 600 & 900 & $0.76521 \pm 0.00022$ \\
\hline $1 \mathrm{~F}$ & Pin $2.1 \mathrm{w} / \mathrm{o} \mathrm{T}_{\mathrm{F}}=900 \mathrm{~K}$ & 600 & 600 & 900 & $1.05835 \pm 0.00017$ \\
\hline $1 \mathrm{G}$ & Pin $3.6 \mathrm{w} / \mathrm{o} \mathrm{T}_{\mathrm{F}}=900 \mathrm{~K}$ & 600 & 600 & 900 & $1.21335 \pm 0.00018$ \\
\hline $1 \mathrm{H}$ & Pin $4.6 \mathrm{w} / \mathrm{o} \mathrm{T}_{\mathrm{F}}=900 \mathrm{~K}$ & 600 & 600 & 900 & $1.27094 \pm 0.00018$ \\
\hline $1 \mathrm{I}$ & Pin Gadolinia rod $\left(5 \% \mathrm{Gd}_{2} \mathrm{O}_{3}\right)$ & 600 & 600 & 900 & $0.21820 \pm 0.00010$ \\
\hline $1 \mathrm{~J}$ & Pin $3.1 \mathrm{w} / \mathrm{o} \mathrm{T}_{\mathrm{F}}=600 / 900 / 1200 \mathrm{~K}$ & 600 & 600 & $600 / 900 / 1200$ & $1.17451 \pm 0.00014$ \\
\hline $2 \mathrm{~A}$ & FA No Poisons $T_{F}=565 K$ & 565 & 565 & 565 & $1.18218 \pm 0.00002$ \\
\hline $2 \mathrm{~B}$ & FA No Poisons $T_{F}=600 K$ & 600 & 600 & 600 & $1.18240 \pm 0.00012$ \\
\hline $2 \mathrm{C}$ & FA No Poisons $T_{F}=900 K$ & 600 & 600 & 900 & $1.17354 \pm 0.00014$ \\
\hline $2 \mathrm{D}$ & FA No Poisons $T_{F}=1200 K$ & 600 & 600 & 1200 & $1.16543 \pm 0.00015$ \\
\hline $2 \mathrm{E}$ & FA 12 Pyrex & 600 & 600 & 900 & $1.06072 \pm 0.00016$ \\
\hline $2 \mathrm{~F}$ & FA 24 Pyrex & 600 & 600 & 900 & $0.96541 \pm 0.00017$ \\
\hline $2 \mathrm{G}$ & FA 24 AIC & 600 & 600 & 900 & $0.83443 \pm 0.00017$ \\
\hline $2 \mathrm{H}$ & FA 24 B4C & 600 & 600 & 900 & $0.77337 \pm 0.00021$ \\
\hline $2 \mathrm{I}$ & FA Instrument Thimble & 600 & 600 & 900 & $1.17256 \pm 0.00012$ \\
\hline $2 \mathrm{~J}$ & FA Instrument +24 Pyrex & 600 & 600 & 900 & $0.96476 \pm 0.00017$ \\
\hline $2 \mathrm{~K}$ & FA Zoned + 24 Pyrex & 600 & 600 & 900 & $1.00886 \pm 0.00019$ \\
\hline $2 \mathrm{~L}$ & FA 80 IFBA & 600 & 600 & 900 & $1.01133 \pm 0.00018$ \\
\hline $2 \mathrm{M}$ & FA 128 IFBA & 600 & 600 & 900 & $0.93205 \pm 0.00017$ \\
\hline $2 \mathrm{~N}$ & FA $104 \mathrm{IFBA}+20 \mathrm{WABA}$ & 600 & 600 & 900 & $0.86123 \pm 0.00020$ \\
\hline $2 \mathrm{O}$ & FA 12 Gadolinia & 600 & 600 & 900 & $1.04004 \pm 0.00017$ \\
\hline $2 \mathrm{P}$ & FA 24 Gadolinia & 600 & 600 & 900 & $0.91993 \pm 0.00016$ \\
\hline
\end{tabular}




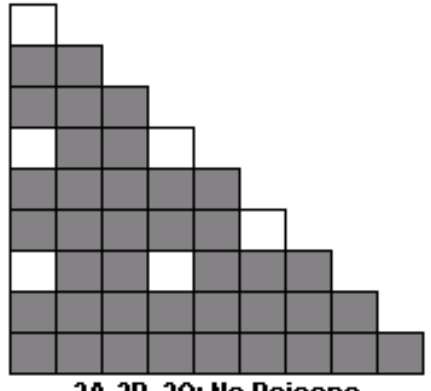

2A-2D, 2Q: Ko Poisons

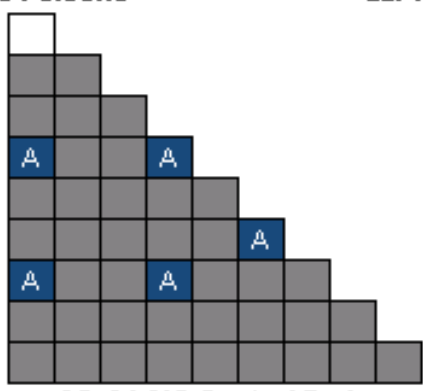

2G: 24 AIC Control Rods

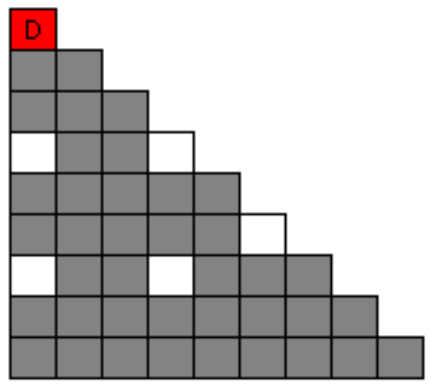

2l: Instrument Thimble

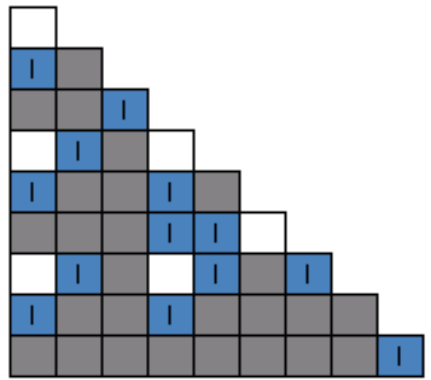

2L: 80 IFBA

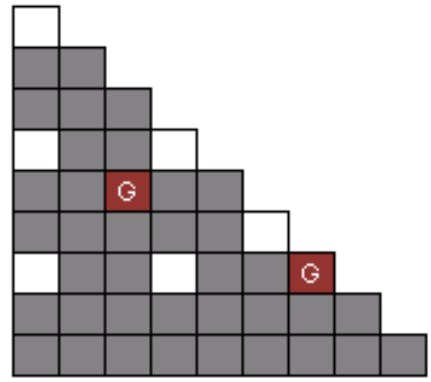

20: 12 Gadolinia

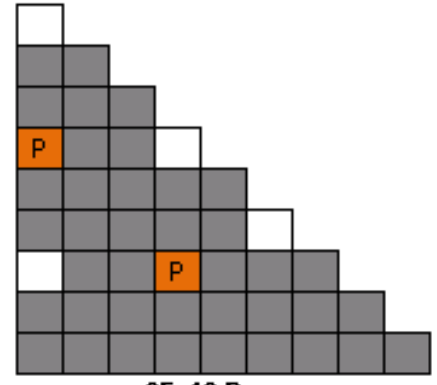

2E: 12 Pyтex

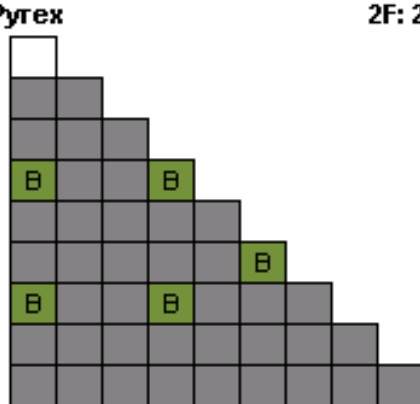

2H: $24 \mathrm{~B}_{4} \mathrm{C}$ Control Rods

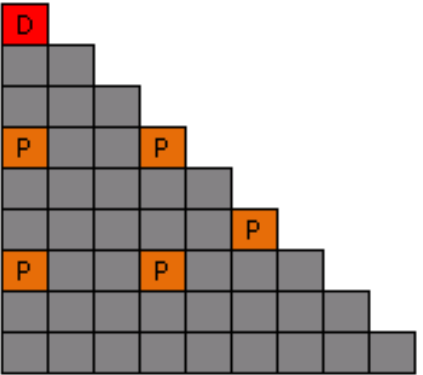

2J: Instrument + 24 Pyiex

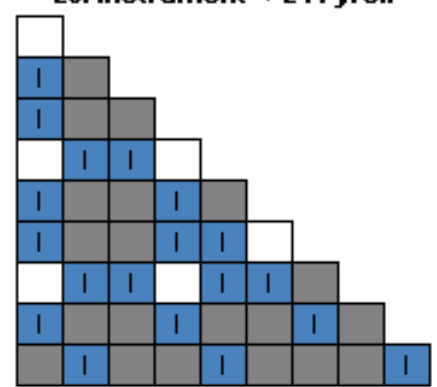

2H: 128 IFBA

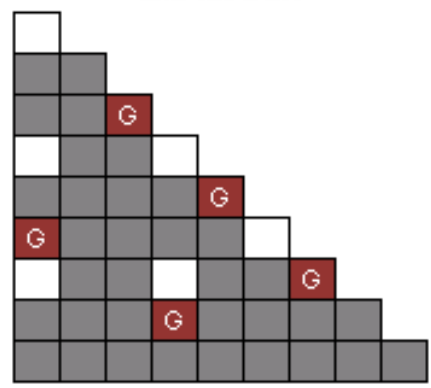

2P: 24 Gadolinia

A AIC Rod

$\mathrm{B} \mathrm{B}_{4} \mathrm{C}$ Rod

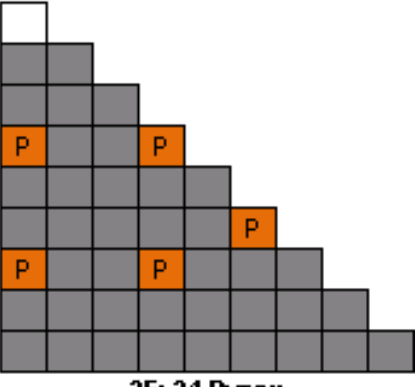

2F: 24 Pyтex
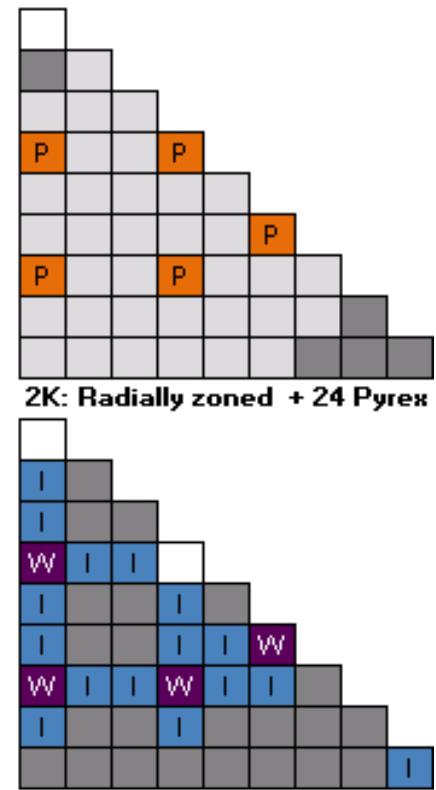

2W: 104 IFBA + 20 WABA

3.1\% Fuel Rod

$3.6 \%$ Fuel Rod

Empty Tube

P Pyrex Rod

Instrument Thimble

I IFBA Fuel Rod

W WABA Fuel Rod

$Q$ Gad - 1.8\% Fuel Rod

Figure 5.1 Lattice pin cell configuration 


\subsection{Calculation options}

The computational results of the depletion calculations would be dependent upon user options and libraries. Therefore, in order not to have any difference due to the differences of user option and library the following user options and library should be utilized in the calculations.

- Xenon option

- Non-equilibrium for all cases

- $\quad$ Equilibrium for the specified cases in Table 5.4 (1C and 2C)

- $\quad$ Power density

- Table 5.1 provides the specific power density for each case $(40.0 \mathrm{w} / \mathrm{gU})$.

- $\quad$ Burnup points

- Table 5.3 provides the burnup points.

- Library

- $\quad$ ENDF/B-7.0 or ENDF/B-7.1 (7.0 is more recommended.)

- The number of depletion zones

- $\mathrm{UO}_{2}$ pellet: 3 equi-volumetric zones

- $\mathrm{UO}_{2}+\mathrm{Gd}_{2} \mathrm{O}_{3}$ Gadolinia rod: 5 equi-volumetric zones

- WABA \& PYREX: 3 equi-volumetric zones

Table 5.3 Burnup points and editing options

\begin{tabular}{|c|c|c|c|c|c|c|c|c|c|c|c|}
\hline \multirow{2}{*}{ Step } & \multicolumn{2}{|c|}{ Burnup } & \multicolumn{3}{|c|}{ Edit } & \multicolumn{3}{c|}{ Burnup } & \multicolumn{3}{c|}{ Edit } \\
\cline { 2 - 8 } & MWD/kgU & EFPD & P.D. & R.R. & Flux & & MWD/kgU & EFPD & P.D. & R.R. & Flux \\
\hline 1 & 0.00 & 0.00 & $\mathrm{O}$ & $\mathrm{O}$ & $\mathrm{O}$ & 21 & 17.00 & 425.00 & & & \\
\hline 2 & 0.01 & 0.25 & & & & 22 & 18.00 & 450.00 & & & \\
\hline 3 & 0.25 & 6.25 & & & & 23 & 19.00 & 475.00 & & & \\
\hline 4 & 0.50 & 12.50 & & & & 24 & 20.00 & 500.00 & O & O & O \\
\hline 5 & 1.00 & 25.00 & & & & 25 & 22.50 & 562.50 & & & \\
\hline 6 & 2.00 & 50.00 & & & & 26 & 25.00 & 625.00 & & & \\
\hline 7 & 3.00 & 75.00 & & & & 27 & 27.50 & 687.50 & & & \\
\hline 8 & 4.00 & 100.00 & & & & 28 & 30.00 & 750.00 & O & O & O \\
\hline 9 & 5.00 & 125.00 & $\mathrm{O}$ & $\mathrm{O}$ & $\mathrm{O}$ & 29 & 32.50 & 812.50 & & & \\
\hline 10 & 6.00 & 150.00 & & & & 30 & 35.00 & 875.00 & & & \\
\hline 11 & 7.00 & 175.00 & & & & 31 & 37.50 & 937.50 & & & \\
\hline 12 & 8.00 & 200.00 & & & & 32 & 40.00 & 1000.00 & $\mathrm{O}$ & $\mathrm{O}$ & $\mathrm{O}$ \\
\hline 13 & 9.00 & 225.00 & & & & 33 & 42.50 & 1062.50 & & & \\
\hline 14 & 10.00 & 250.00 & $\mathrm{O}$ & $\mathrm{O}$ & $\mathrm{O}$ & 34 & 45.00 & 1125.00 & & & \\
\hline 15 & 11.00 & 275.00 & & & & 35 & 47.50 & 1187.50 & & & \\
\hline 16 & 12.00 & 300.00 & & & & 36 & 50.00 & 1250.00 & $\mathrm{O}$ & $\mathrm{O}$ & $\mathrm{O}$ \\
\hline 17 & 13.00 & 325.00 & & & & 37 & 52.50 & 1312.50 & & & \\
\hline 18 & 14.00 & 350.00 & & & & 38 & 55.00 & 1375.00 & & & \\
\hline 19 & 15.00 & 375.00 & $\mathrm{O}$ & $\mathrm{O}$ & $\mathrm{O}$ & 39 & 57.50 & 1437.50 & & & \\
\hline 20 & 16.00 & 400.00 & & & & 40 & 60.00 & 1500.00 & & & \\
\hline
\end{tabular}

P.D. : Power Distribution

R.R. : Reaction Rate 


\subsection{Edition of reactor physics parameters}

In order to complete the code-to-code comparison the following parameters need to be edited which are also provided in Table 5.3.

- Multiplication factors (keff) vs. burnup

- All cases

- All burnup points

- $\quad$ Pin power distribution

- $\quad$ For the problems: $\mathbf{2 C}, \mathbf{2 F}, \mathbf{2 N}$ and $\mathbf{2 P}$

- For the burnup points: 1, 8, 13, 18, 23, 27, 31 and 35

- $\quad$ Particle(atomic) number densities (PND)

- $\quad$ For the problems: 1C, 1E and 1H

- PND should be edited for each depletion zone. (e.g. UO2 pellet=3 zones)

- $\quad$ PND needs to be edited for the nuclides in Table 4.1.

- All burnup points

- Multigroup scalar fluxes

- For the problems: $\mathbf{1 C}$ and $\mathbf{1 H}$

- For the burnup points: 1, 8, 13, 18, 23, 27, 31 and 35

- Power scaled 1-group scalar flux for each depletion zone

- Monte Carlo codes: Multi-group (47-group) normalized scalar flux per unit lethargy. (The 47-g structure is provided on Table 5.5.)

- Deterministic codes: Multi-group normalized scalar flux per unit lethargy with their own group structure.

- $\quad$ Reaction rates

- For the problems: $\mathbf{1 C}$ and $\mathbf{1 H}$

- For the burnup points: 1, 8, 13, 18, 23, 27, 31 and 35

- $\quad$ 1-group absorption and fission reaction rates for each depletion zone

- For nuclides: Table 5.6 provides the list of nuclides

\subsection{Sensitivities}

Sensitivity calculations need to be performed fot the following parameters.

- $\underline{\text { Xenon }}$

- For the problems: 1C and 2C

- All burnup points

- Equilibrium xenon option

- $\underline{X e n o n}$

- For the problems: $\mathbf{1 C}$ and $\mathbf{2 C}$ 
- All burnup points

- Equilibrium xenon option

- Burnup intervals

- For the problems: 1C

- All burnup points

- Ver fine burnup points: up to participants

- Number of intra depletion zones for gadolinia rod

- For the problems: 1H

- All burnup points

- 10 intra depletion zones

Table 5.4 Xenon option and editing parameters

\begin{tabular}{|c|c|c|c|c|c|c|c|c|c|c|}
\hline \multirow[b]{2}{*}{ No } & \multirow[b]{2}{*}{ Problem } & \multirow[b]{2}{*}{ Description } & \multicolumn{2}{|c|}{ Xenon } & \multirow{2}{*}{$\begin{array}{l}\text { Special } \\
\text { option }\end{array}$} & \multicolumn{5}{|c|}{ Edit } \\
\hline & & & $\begin{array}{l}\text { Tran- } \\
\text { sient }\end{array}$ & $\begin{array}{l}\text { Equi- } \\
\text { librium }\end{array}$ & & $\mathrm{k}_{\mathrm{eff}}$ & $\begin{array}{c}\text { Pin } \\
\text { power }\end{array}$ & PND & $\begin{array}{c}\text { Reaction } \\
\text { rate }\end{array}$ & Flux \\
\hline 1 & $1 \mathrm{~A}$ & Pin $3.1 \mathrm{w} / \mathrm{o} \mathrm{T}_{\mathrm{F}}=565 \mathrm{~K}$ & $\mathrm{O}$ & & & $\mathrm{O}$ & & & & \\
\hline 2 & 1B & Pin $3.1 \mathrm{w} / \mathrm{o} \mathrm{T}_{\mathrm{F}}=600 \mathrm{~K}$ & $\mathrm{O}$ & & & $\mathrm{O}$ & & & & \\
\hline 3 & $1 \mathrm{C}$ & Pin $3.1 \mathrm{w} / \mathrm{o} \mathrm{T}_{\mathrm{F}}=900 \mathrm{~K}$ & $\mathrm{O}$ & $\mathrm{O}$ & $* \mathrm{~A}$ & $\mathrm{O}$ & & $\mathrm{O}$ & $\mathrm{O}$ & $\mathrm{O}$ \\
\hline 4 & 1D & Pin $3.1 \mathrm{w} / \mathrm{o} \mathrm{T}_{\mathrm{F}}=1200 \mathrm{~K}$ & $\mathrm{O}$ & & & $\mathrm{O}$ & & & & \\
\hline 5 & $1 \mathrm{E}$ & Pin IFBA $3.1 \mathrm{w} / \mathrm{o} \mathrm{T}_{\mathrm{F}}=565 \mathrm{~K}$ & $\mathrm{O}$ & & & $\mathrm{O}$ & & $\mathrm{O}$ & & \\
\hline 6 & $1 \mathrm{~F}$ & Pin $3.6 \mathrm{w} / \mathrm{o} \mathrm{T}_{\mathrm{F}}=900 \mathrm{~K}$ & $\mathrm{O}$ & & & $\mathrm{O}$ & & & & \\
\hline 7 & $1 G$ & Pin $3.6 \mathrm{w} / \mathrm{o} \mathrm{T}_{\mathrm{F}}=900 \mathrm{~K}$ & $\mathrm{O}$ & & & $\mathrm{O}$ & & & & \\
\hline 8 & $1 \mathrm{H}$ & Pin Gadolinia rod $\left(5 \% \mathrm{Gd}_{2} \mathrm{O}_{3}\right)$ & $\mathrm{O}$ & & $* \mathrm{~B}$ & $\mathrm{O}$ & & $\mathrm{O}$ & $\mathrm{O}$ & $\mathrm{O}$ \\
\hline 9 & $1 \mathrm{I}$ & Pin $3.1 \mathrm{w} / \mathrm{o} \mathrm{T}_{\mathrm{F}}=600 / 900 / 1200 \mathrm{~K}$ & $\mathrm{O}$ & & & $\mathrm{O}$ & & & & \\
\hline 10 & $2 \mathrm{~A}$ & FA No Poisons $T_{F}=565 K$ & $\mathrm{O}$ & & & $\mathrm{O}$ & & & & \\
\hline 11 & 2B & FA No Poisons $T_{F}=600 K$ & $\mathrm{O}$ & & & $\mathrm{O}$ & & & & \\
\hline 12 & $2 \mathrm{C}$ & FA No Poisons $T_{F}=900 K$ & $\mathrm{O}$ & $\mathrm{O}$ & & $\mathrm{O}$ & $\mathrm{O}$ & & & \\
\hline 13 & $2 \mathrm{D}$ & FA No Poisons $T_{F}=1200 K$ & $\mathrm{O}$ & & & $\mathrm{O}$ & & & & \\
\hline 14 & $2 \mathrm{E}$ & FA 12 Pyrex & $\mathrm{O}$ & & & $\mathrm{O}$ & & & & \\
\hline 15 & $2 \mathrm{~F}$ & FA 24 Pyrex & $\mathrm{O}$ & & & $\mathrm{O}$ & $\mathrm{O}$ & & & \\
\hline 16 & $2 \mathrm{G}$ & FA 24 AIC & $\mathrm{O}$ & & & $\mathrm{O}$ & & & & \\
\hline 17 & $2 \mathrm{H}$ & FA 24 B4C & $\mathrm{O}$ & & & $\mathrm{O}$ & & & & \\
\hline 18 & $2 \mathrm{I}$ & FA Instrument Thimble & $\mathrm{O}$ & & & $\mathrm{O}$ & & & & \\
\hline 19 & $2 \mathrm{~J}$ & FA Instrument + 24 Pyrex & $\mathrm{O}$ & & & $\mathrm{O}$ & & & & \\
\hline 20 & $2 \mathrm{~K}$ & FA Zoned + 24 Pyrex & $\mathrm{O}$ & & & $\mathrm{O}$ & & & & \\
\hline 21 & $2 \mathrm{~L}$ & FA 80 IFBA & $\mathrm{O}$ & & & $\mathrm{O}$ & & & & \\
\hline 22 & $2 \mathrm{M}$ & FA 128 IFBA & $\mathrm{O}$ & & & $\mathrm{O}$ & & & & \\
\hline 23 & $2 \mathrm{~N}$ & FA $104 \mathrm{IFBA}+20 \mathrm{WABA}$ & $\mathrm{O}$ & & & $\mathrm{O}$ & $\mathrm{O}$ & & & \\
\hline 24 & $2 \mathrm{O}$ & FA 12 Gadolinia & $\mathrm{O}$ & & & $\mathrm{O}$ & & & & \\
\hline 25 & $2 \mathrm{P}$ & FA 24 Gadolinia & $\mathrm{O}$ & & & $\mathrm{O}$ & $\mathrm{O}$ & & & \\
\hline
\end{tabular}

*A: Burnup interval sensitivy calculation

*B: 10 intra pin subzones for gadolinia rod 
Table 5.5 The 47-group boundaries

\begin{tabular}{|c|c|c|c|c|c|c|c|c|c|}
\hline Gr & Upper $(\mathrm{eV})$ & $\mathrm{Gr}$ & Upper $(\mathrm{eV})$ & $\mathrm{Gr}$ & Upper $(\mathrm{eV})$ & $\mathrm{Gr}$ & Upper $(\mathrm{eV})$ & $\mathrm{Gr}$ & Upper $(\mathrm{eV})$ \\
\hline 1 & $2.00000 \mathrm{E}+07$ & 11 & $2.03470 \mathrm{E}+03$ & 21 & $5.71501 \mathrm{E}+00$ & 31 & $1.07220 \mathrm{E}+00$ & 41 & $1.45721 \mathrm{E}-01$ \\
\hline 2 & $6.06530 \mathrm{E}+06$ & 12 & $1.30070 \mathrm{E}+02$ & 22 & $5.04348 \mathrm{E}+00$ & 32 & $1.01370 \mathrm{E}+00$ & 42 & $1.11570 \mathrm{E}-01$ \\
\hline 3 & $3.67880 \mathrm{E}+06$ & 13 & $7.88933 \mathrm{E}+01$ & 23 & $4.45090 \mathrm{E}+00$ & 33 & $9.71004 \mathrm{E}-01$ & 43 & $8.19682 \mathrm{E}-02$ \\
\hline 4 & $2.23130 \mathrm{E}+06$ & 14 & $4.78512 \mathrm{E}+01$ & 24 & $3.92790 \mathrm{E}+00$ & 34 & $9.09997 \mathrm{E}-01$ & 44 & $5.69219 \mathrm{E}-02$ \\
\hline 5 & $1.35340 \mathrm{E}+06$ & 15 & $2.90229 \mathrm{E}+01$ & 25 & $2.38239 \mathrm{E}+00$ & 35 & $7.82083 \mathrm{E}-01$ & 45 & $4.27552 \mathrm{E}-02$ \\
\hline 6 & $8.20850 \mathrm{E}+05$ & 16 & $1.37100 \mathrm{E}+01$ & 26 & $1.85539 \mathrm{E}+00$ & 36 & $6.25062 \mathrm{E}-01$ & 46 & $3.06129 \mathrm{E}-02$ \\
\hline 7 & $4.97870 \mathrm{E}+05$ & 17 & $1.20990 \mathrm{E}+01$ & 27 & $1.45740 \mathrm{E}+00$ & 37 & $5.03232 \mathrm{E}-01$ & 47 & $1.23960 \mathrm{E}-02$ \\
\hline 8 & $1.83160 \mathrm{E}+05$ & 18 & $8.31529 \mathrm{E}+00$ & 28 & $1.23511 \mathrm{E}+00$ & 38 & $3.57670 \mathrm{E}-01$ & & $1.00000 \mathrm{E}-05$ \\
\hline 9 & $6.73790 \mathrm{E}+04$ & 19 & $7.33822 \mathrm{E}+00$ & 29 & $1.16640 \mathrm{E}+00$ & 39 & $2.70521 \mathrm{E}-01$ & & \\
\hline 10 & $9.11880 \mathrm{E}+03$ & 20 & $6.47602 \mathrm{E}+00$ & 30 & $1.12540 \mathrm{E}+00$ & 40 & $1.84430 \mathrm{E}-01$ & & \\
\hline
\end{tabular}

Table 5.6 Nuclides for editing reaction rates

\begin{tabular}{|c|c|c|c|c|c|c|c|}
\hline ID & Nuclide & ID & Nuclide & ID & Nuclide & ID & Nuclide \\
\hline 42095 & ${ }^{95} \mathrm{Mo}$ & 54135 & ${ }^{135} \mathrm{Xe}$ & 61148 & ${ }^{148} \mathrm{Pm}$ & 94238 & ${ }^{238} \mathrm{Pu}$ \\
\hline 43099 & ${ }^{99} \mathrm{Tc}$ & 55133 & ${ }^{133} \mathrm{Cs}$ & 62149 & ${ }^{149} \mathrm{Sm}$ & 94239 & ${ }^{239} \mathrm{Pu}$ \\
\hline 44101 & ${ }^{101} \mathrm{Ru}$ & 55134 & ${ }^{133} \mathrm{Cs}$ & 63155 & ${ }^{155} \mathrm{Eu}$ & 94240 & ${ }^{240} \mathrm{Pu}$ \\
\hline 45103 & ${ }^{103} \mathrm{Rh}$ & 60143 & ${ }^{143} \mathrm{Nd}$ & 92235 & ${ }^{235} \mathrm{U}$ & 94241 & ${ }^{241} \mathrm{Pu}$ \\
\hline 46105 & ${ }^{105} \mathrm{Pd}$ & 60145 & ${ }^{145} \mathrm{Nd}$ & 92236 & ${ }^{236} \mathrm{U}$ & 94242 & ${ }^{242} \mathrm{Pu}$ \\
\hline 46107 & ${ }^{107} \mathrm{Pd}$ & 62150 & ${ }^{150} \mathrm{Sm}$ & 92238 & ${ }^{238} \mathrm{U}$ & 95243 & ${ }^{243} \mathrm{Am}$ \\
\hline 46108 & ${ }^{108} \mathrm{Pd}$ & 62151 & ${ }^{150} \mathrm{Sm}$ & 93237 & ${ }^{237} \mathrm{~Np}$ & & \\
\hline
\end{tabular}




\section{DISCUSSION}

Currently the programs in Table 6.1 are supposed to be engaged in this code-to-code comparison for the depletion benchmark calculations.

Table 6.1 Transport depletion codes

\begin{tabular}{|c|c|c|c|c|c|}
\hline \multirow{2}{*}{ Code } & \multicolumn{3}{|c|}{ Solver } & \multirow{2}{*}{ Developer } & \multirow{2}{*}{ Group } \\
\cline { 2 - 4 } & Transport & Depletion & Resonance & & \\
\hline MPACT & MOC & ORIGEN & Subgroup & CASL & $47 / 51$ \\
\hline SHIFT & Monte Carlo & ORIGEN & & ORNL & CE \\
\hline POLARIS & MOC & ORIGEN & ESSM & ORNL & $252 / 56$ \\
\hline TRITON & Monte Carlo & ORIGEN & CENTRM & ORNL & $252 / 56$ \\
\hline nTRACER & MOC & Krylov & Subgroup & SNU & 47 \\
\hline McCARD & Monte Carlo & ORIGEN & & SNU & CE \\
\hline STREAM & MOC & & Equivalence & UNIST & MG \\
\hline SERPENT & Monte Carlo & CRAM & & VTT & CE \\
\hline MCNP6 & Monte Carlo & & & LANL & CE \\
\hline
\end{tabular}

*ORIGEN: Exponential matrix + Bateman

\section{ACKNOWLEDGEMENT}

The author would like to acknowledge Kevin T. Clarno who is the CASL PHI lead for his efforts to review this document in detail and provide valuable comments. 


\section{REFERENCE}

[God14] Andrew T. Godfrey, "VERA Core Physics Benchmark Progression Problem Specifications," CASL-U-2012-0131-004, Rev. 4 (8/29/2014).

[Wie14] W. Wieselquist, K. S. Kim , "Simplified Burnup Chain Development," CASL PHI Ticket\#3432 (9/10/2014). 
Appendix A. Constituent nuclides for the simplified burnup chain

\begin{tabular}{|c|c|c|c|c|c|}
\hline No & Nuclide & CAT & ID & IFPY & Special Yield Definition $\left(\mathrm{ID}_{\mathrm{i}} / \mathrm{W}_{\mathrm{i}} / \mathrm{IFPY}_{\mathrm{i}}\right)$ \\
\hline 1 & $5-\mathrm{B}-0$ & 1 & 50100 & 0 & \\
\hline 2 & $5-B-10$ & 1 & 50110 & 0 & \\
\hline 3 & $8-0-16$ & 1 & 80160 & 0 & \\
\hline 4 & $47-\mathrm{Ag}-107$ & 1 & 471070 & 0 & \\
\hline 5 & 47-Ag-109 & 1 & 471090 & 0 & \\
\hline 6 & $47-\mathrm{Ag}-110$ & 1 & 471100 & 0 & \\
\hline 7 & $48-\mathrm{Cd}-110$ & 1 & 481100 & 0 & \\
\hline 8 & 48-Cd-111 & 1 & 481110 & 0 & \\
\hline 9 & $48-\mathrm{Cd}-112$ & 1 & 481120 & 0 & \\
\hline 10 & 48-Cd-113 & 1 & 481130 & 0 & \\
\hline 11 & $48-\mathrm{Cd}-114$ & 1 & 481140 & 0 & \\
\hline 12 & $48-\mathrm{Cd}-115$ & 1 & 481150 & 0 & \\
\hline 13 & 49-In-113 & 1 & 491130 & 0 & \\
\hline 14 & $49-I n-115$ & 1 & 491150 & 0 & \\
\hline 15 & $62-\mathrm{Sm}-152$ & 1 & 621520 & 0 & \\
\hline 16 & $62-\mathrm{Sm}-153$ & 1 & 621530 & 0 & \\
\hline 17 & 63-Eu-151 & 1 & 631510 & 0 & \\
\hline 18 & 63-Eu-152 & 1 & 631520 & 0 & \\
\hline 19 & 63-Eu-152m & 1 & 631521 & 0 & \\
\hline 20 & 63-Eu-153 & 1 & 631530 & 0 & \\
\hline 21 & 63-Eu-154 & 1 & 631540 & 0 & \\
\hline 22 & 63-Eu-155 & 1 & 631550 & 0 & \\
\hline 23 & 63-Eu-156 & 1 & 631560 & 0 & \\
\hline 24 & 63-Eu-157 & 1 & 631570 & 0 & \\
\hline 25 & 64-Gd-152 & 1 & 641520 & 0 & \\
\hline 26 & 64-Gd-154 & 1 & 641540 & 0 & \\
\hline 27 & 64-Gd-155 & 1 & 641550 & 0 & \\
\hline 28 & 64-Gd-156 & 1 & 641560 & 0 & \\
\hline 29 & 64-Gd-157 & 1 & 641570 & 0 & \\
\hline 30 & 64-Gd-158 & 1 & 641580 & 0 & \\
\hline 31 & 64-Gd-159 & 1 & 641590 & 0 & \\
\hline 32 & 64-Gd-160 & 1 & 641600 & 0 & \\
\hline 33 & 64-Gd-161 & 1 & 641610 & 0 & \\
\hline 34 & $65-\mathrm{Tb}-159$ & 1 & 651590 & 0 & \\
\hline 35 & $65-\mathrm{Tb}-160$ & 1 & 651600 & 0 & \\
\hline 36 & $65-\mathrm{Tb}-161$ & 1 & 651610 & 0 & \\
\hline 37 & 66-Dy-160 & 1 & 661600 & 0 & \\
\hline 38 & 66-Dy-161 & 1 & 661610 & 0 & \\
\hline 39 & 66-Dy-162 & 1 & 661620 & 0 & \\
\hline 40 & 66-Dy-163 & 1 & 661630 & 0 & \\
\hline 41 & 66-Dy-164 & 1 & 661640 & 0 & \\
\hline 42 & 66-Dy-165 & 1 & 661650 & 0 & \\
\hline 43 & 67-Нo-165 & 1 & 671650 & 0 & \\
\hline 44 & 68-Er-162 & 1 & 681620 & 0 & \\
\hline 45 & 68-Er-164 & 1 & 681640 & 0 & \\
\hline 46 & 68-Er-166 & 1 & 681660 & 0 & \\
\hline 47 & 68-Er-167 & 1 & 681670 & 0 & \\
\hline 48 & 68-Er-168 & 1 & 681680 & 0 & \\
\hline 49 & 68-Er-169 & 1 & 681690 & 0 & \\
\hline 50 & $68-\mathrm{Er}-170$ & 1 & 681700 & 0 & \\
\hline
\end{tabular}




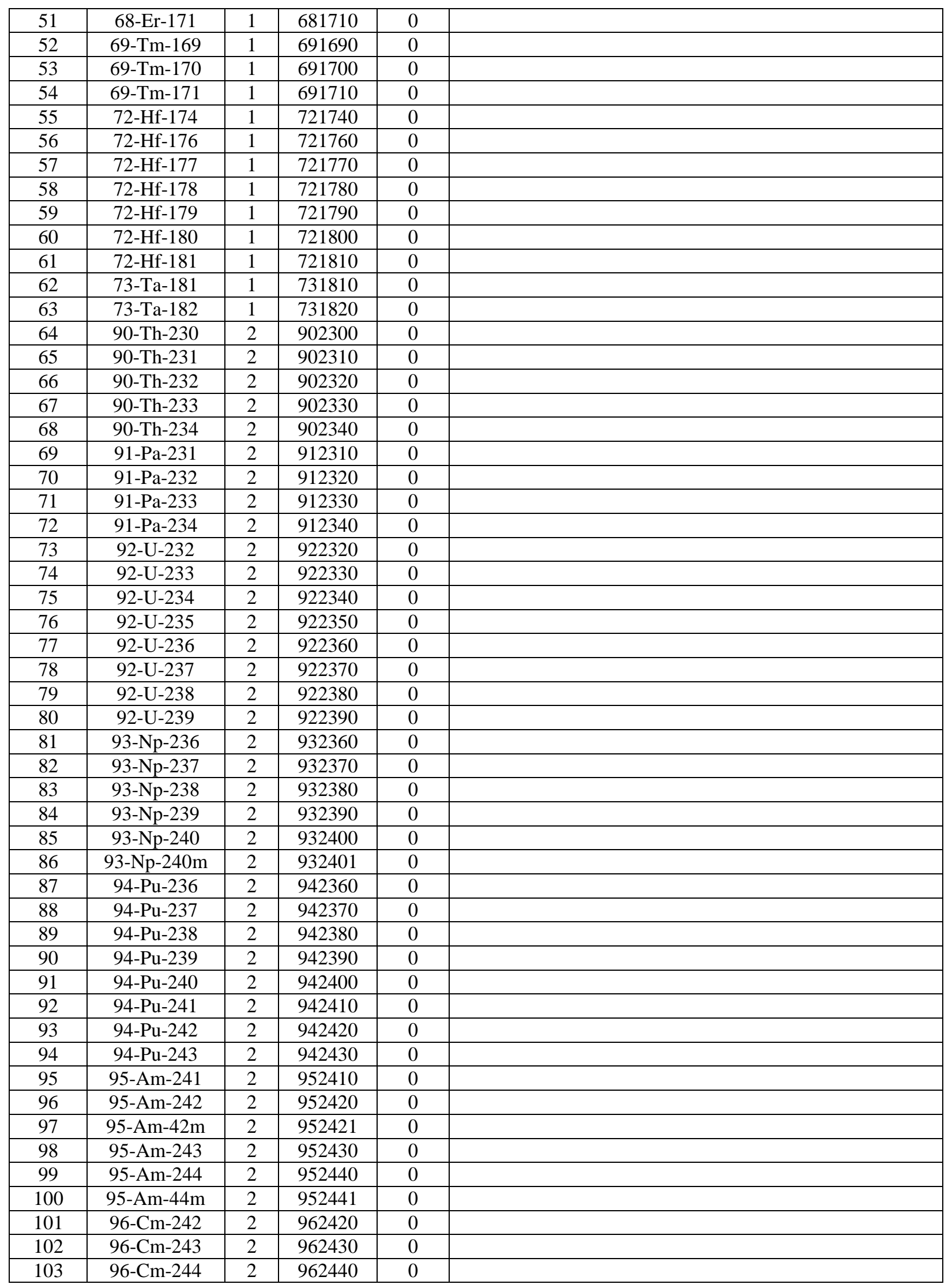




\begin{tabular}{|c|c|c|c|c|c|}
\hline 104 & 96-Cm-245 & 2 & 962450 & 0 & \\
\hline 105 & $96-\mathrm{Cm}-246$ & 2 & 962460 & 0 & \\
\hline 106 & $35-\mathrm{BR}-81$ & 3 & 350810 & 2 & \\
\hline 107 & $35-\mathrm{BR}-82$ & 3 & 350820 & 2 & \\
\hline 108 & $36-\mathrm{KR}-82$ & 3 & 360820 & 3 & $350821 / 0.024 / 1, \quad 360820 / 1.000 / 1$ \\
\hline 109 & $36-\mathrm{KR}-83$ & 3 & 360830 & 2 & \\
\hline 110 & $36-\mathrm{KR}-84$ & 3 & 360840 & 2 & \\
\hline 111 & $36-\mathrm{KR}-85$ & 3 & 360850 & 2 & \\
\hline 112 & $36-\mathrm{KR}-86$ & 3 & 360860 & 2 & \\
\hline 113 & 38-SR-89 & 3 & 380890 & 2 & \\
\hline 114 & 38-SR-90 & 3 & 380900 & 2 & \\
\hline 115 & $39-\mathrm{Y}-89$ & 3 & 390890 & 1 & \\
\hline 116 & $39-\mathrm{Y}-90$ & 3 & 390900 & 1 & \\
\hline 117 & $39-Y-91$ & 3 & 390910 & 2 & \\
\hline 118 & 40-ZR-91 & 3 & 400910 & 1 & \\
\hline 119 & 40-ZR-93 & 3 & 400930 & 2 & \\
\hline 120 & $40-Z R-95$ & 3 & 400950 & 2 & \\
\hline 121 & 40-ZR-96 & 3 & 400960 & 2 & \\
\hline 122 & $41-N B-95$ & 3 & 410950 & 3 & $410950 / 1.000 / 1, \quad 410951 / 0.944 / 1$ \\
\hline 123 & $42-\mathrm{MO}-95$ & 3 & 420950 & 3 & $410951 / 0.056 / 1, \quad 420950 / 1.000 / 1$ \\
\hline 124 & 42-MO-96 & 3 & 420960 & 3 & $410960 / 1.000 / 1, \quad 420960 / 1.000 / 1$ \\
\hline 125 & 42-MO-97 & 3 & 420970 & 2 & \\
\hline 126 & 42-MO-98 & 3 & 420980 & 2 & \\
\hline 127 & 42-MO-99 & 3 & 420990 & 2 & \\
\hline 128 & 42-MO-100 & 3 & 421000 & 2 & \\
\hline 129 & 43-TC-99 & 3 & 430990 & 1 & \\
\hline 130 & 43-TC-99m & 3 & 430991 & 1 & \\
\hline 131 & 43-TC-100 & 3 & 431000 & 1 & \\
\hline 132 & $44-R U-100$ & 3 & 441000 & 1 & \\
\hline 133 & 44-RU-101 & 3 & 441010 & 2 & \\
\hline 134 & $44-R U-102$ & 3 & 441020 & 2 & \\
\hline 135 & $44-R U-103$ & 3 & 441030 & 2 & \\
\hline 136 & 44-RU-104 & 3 & 441040 & 2 & \\
\hline 137 & 44-RU-105 & 3 & 441050 & 2 & \\
\hline 138 & $44-R U-106$ & 3 & 441060 & 2 & \\
\hline 139 & 45-RH-102 & 3 & 451020 & 1 & \\
\hline 140 & $45-\mathrm{RH}-102 \mathrm{~m}$ & 3 & 451021 & 1 & \\
\hline 141 & 45-RH-103 & 3 & 451030 & 1 & \\
\hline 142 & 45-RH-103m & 3 & 451031 & 1 & \\
\hline 143 & 45-RH-104 & 3 & 451040 & 1 & \\
\hline 144 & 45-RH-105 & 3 & 451050 & 1 & \\
\hline 145 & 45-RH-105m & 3 & 451051 & 1 & \\
\hline 146 & 45-RH-106 & 3 & 451060 & 1 & \\
\hline 147 & 45-RH-106m & 3 & 451061 & 1 & \\
\hline 148 & 46-PD-104 & 3 & 461040 & 1 & \\
\hline 149 & 46-PD-105 & 3 & 461050 & 1 & \\
\hline 150 & 46-PD-106 & 3 & 461060 & 1 & \\
\hline 151 & 46-PD-107 & 3 & 461070 & 2 & \\
\hline 152 & 46-PD-108 & 3 & 461080 & 2 & \\
\hline 153 & 46-PD-109 & 3 & 461090 & 2 & \\
\hline 154 & 47-AG-109 & 3 & 471090 & 1 & \\
\hline 155 & 47-AG-109m & 3 & 471091 & 1 & \\
\hline 156 & 47-AG-110 & 3 & 471100 & 2 & \\
\hline
\end{tabular}




\begin{tabular}{|c|c|c|c|c|c|}
\hline 157 & 47-AG-110m & 3 & 471101 & 2 & \\
\hline 158 & 47-AG-111 & 3 & 471110 & 2 & \\
\hline 159 & $48-C D-110$ & 3 & 481100 & 1 & \\
\hline 160 & 48-CD-111 & 3 & 481110 & 3 & $471110 /-1.000 / 2, \quad 481110 / 1.000 / 2, \quad 481111 / 1.000 / 1$ \\
\hline 161 & $48-C D-113$ & 3 & 481130 & 2 & \\
\hline 162 & $49-\mathrm{IN}-115$ & 3 & 491150 & 2 & \\
\hline 163 & 51-SB-121 & 3 & 511210 & 2 & \\
\hline 164 & $51-\mathrm{SB}-123$ & 3 & 511230 & 2 & \\
\hline 165 & $51-\mathrm{SB}-125$ & 3 & 511250 & 2 & \\
\hline 166 & 51-SB-127 & 3 & 511270 & 2 & \\
\hline 167 & 52-TE-127 & 3 & 521270 & -1 & \\
\hline 168 & $52-\mathrm{TE}-127 \mathrm{~m}$ & 3 & 521271 & -1 & \\
\hline 169 & 52-TE-129m & 3 & 521291 & 2 & \\
\hline 170 & 52-TE-132 & 3 & 521320 & 2 & \\
\hline 171 & $53-\mathrm{I}-127$ & 3 & 531270 & 1 & \\
\hline 172 & $53-\mathrm{I}-128$ & 3 & 531280 & 3 & $531280 / 0.931 / 2$ \\
\hline 173 & $53-\mathrm{I}-129$ & 3 & 531290 & 3 & $531290 / 1.000 / 2, \quad 521291 /-1.000 / 2$ \\
\hline 174 & $53-\mathrm{I}-130$ & 3 & 531300 & 2 & \\
\hline 175 & 53-I-131 & 3 & 531310 & 2 & \\
\hline 176 & $53-\mathrm{I}-132$ & 3 & 531320 & 1 & \\
\hline 177 & $53-\mathrm{I}-135$ & 3 & 531350 & 2 & \\
\hline 178 & 54-XE-128 & 3 & 541280 & 1 & \\
\hline 179 & 54-XE-130 & 3 & 541300 & 1 & \\
\hline 180 & 54-XE-131 & 3 & 541310 & 1 & \\
\hline 181 & 54-XE-132 & 3 & 541320 & 1 & \\
\hline 182 & 54-XE-133 & 3 & 541330 & 2 & \\
\hline 183 & 54-XE-134 & 3 & 541340 & 2 & \\
\hline 184 & 54-XE-135 & 3 & 541350 & 1 & \\
\hline 185 & 54-XE-135m & 3 & 541351 & 1 & \\
\hline 186 & 54-XE-136 & 3 & 541360 & 2 & \\
\hline 187 & 54-XE-137 & 3 & 541370 & 2 & \\
\hline 188 & 55-CS-133 & 3 & 551330 & 1 & \\
\hline 189 & 55-CS-134 & 3 & 551340 & 1 & \\
\hline 190 & 55-CS-135 & 3 & 551350 & 1 & \\
\hline 191 & 55-CS-136 & 3 & 551360 & 1 & \\
\hline 192 & 55-CS-137 & 3 & 551370 & 1 & \\
\hline 193 & 56-BA-134 & 3 & 561340 & 1 & \\
\hline 194 & 56-BA-137 & 3 & 561370 & 1 & \\
\hline 195 & 56-BA-140 & 3 & 561400 & 2 & \\
\hline 196 & 57-LA-139 & 3 & 571390 & 2 & \\
\hline 197 & 57-LA-140 & 3 & 571400 & 1 & \\
\hline 198 & 58-CE-140 & 3 & 581400 & 1 & \\
\hline 199 & 58-CE-141 & 3 & 581410 & 2 & \\
\hline 200 & 58-CE-142 & 3 & 581420 & 2 & \\
\hline 201 & 58-CE-143 & 3 & 581430 & 2 & \\
\hline 202 & 58-CE-144 & 3 & 581440 & 2 & \\
\hline 203 & 59-PR-141 & 3 & 591410 & 1 & \\
\hline 204 & 59-PR-142 & 3 & 591420 & 1 & \\
\hline 205 & 59-PR-143 & 3 & 591430 & 1 & \\
\hline 206 & 59-PR-144 & 3 & 591440 & 1 & \\
\hline 207 & 60-ND-142 & 3 & 601420 & 1 & \\
\hline 208 & 60-ND-143 & 3 & 601430 & 1 & \\
\hline 209 & 60-ND-144 & 3 & 601440 & 1 & \\
\hline
\end{tabular}




\begin{tabular}{|c|c|c|c|c|c|}
\hline 210 & 60-ND-145 & 3 & 601450 & 2 & \\
\hline 211 & 60-ND-146 & 3 & 601460 & 2 & \\
\hline 212 & 60-ND-147 & 3 & 601470 & 2 & \\
\hline 213 & 60-ND-148 & 3 & 601480 & 2 & \\
\hline 214 & 60-ND-149 & 3 & 601490 & 2 & \\
\hline 215 & 60-ND-150 & 3 & 601500 & 2 & \\
\hline 216 & 60-ND-151 & 3 & 601510 & 2 & \\
\hline 217 & 61-PM-147 & 3 & 611470 & 1 & \\
\hline 218 & 61-PM-148 & 3 & 611480 & -1 & \\
\hline 219 & 61-PM-148m & 3 & 611481 & -1 & \\
\hline 220 & 61-PM-149 & 3 & 611490 & 1 & \\
\hline 221 & 61-PM-150 & 3 & 611500 & 1 & \\
\hline 222 & 61-PM-151 & 3 & 611510 & 1 & \\
\hline 223 & 62-SM-147 & 3 & 621470 & 1 & \\
\hline 224 & 62-SM-148 & 3 & 621480 & 1 & \\
\hline 225 & 62-SM-149 & 3 & 621490 & 1 & \\
\hline 226 & $62-\mathrm{SM}-150$ & 3 & 621500 & 1 & \\
\hline 227 & 62-SM-151 & 3 & 621510 & 1 & \\
\hline 228 & 62-SM-152 & 3 & 621520 & 2 & \\
\hline 229 & 62-SM-153 & 3 & 621530 & 2 & \\
\hline 230 & 62-SM-154 & 3 & 621540 & 2 & \\
\hline 231 & 62-SM-155 & 3 & 621550 & 2 & \\
\hline 232 & 63-EU-151 & 3 & 631510 & 1 & \\
\hline 233 & 63-EU-153 & 3 & 631530 & 1 & \\
\hline 234 & 63-EU-154 & 3 & 631540 & 1 & \\
\hline 235 & 63-EU-155 & 3 & 631550 & 1 & \\
\hline 236 & 63-EU-156 & 3 & 631560 & 2 & \\
\hline 237 & 63-EU-157 & 3 & 631570 & 2 & \\
\hline 238 & 64-GD-154 & 3 & 641540 & 1 & \\
\hline 239 & 64-GD-155 & 3 & 641550 & 1 & \\
\hline 240 & 64-GD-156 & 3 & 641560 & 1 & \\
\hline 241 & 64-GD-157 & 3 & 641570 & 1 & \\
\hline 242 & 64-GD-158 & 3 & 641580 & 2 & \\
\hline 243 & 64-GD-159 & 3 & 641590 & 2 & \\
\hline 244 & 64-GD-160 & 3 & 641600 & 2 & \\
\hline 245 & 64-GD-161 & 3 & 641610 & 2 & \\
\hline 246 & 65-TB-159 & 3 & 651590 & 1 & \\
\hline 247 & $65-\mathrm{TB}-160$ & 3 & 651600 & 1 & \\
\hline 248 & 65-TB-161 & 3 & 651610 & 1 & \\
\hline 249 & 66-DY-160 & 3 & 661600 & 1 & \\
\hline 250 & 66-DY-161 & 3 & 661610 & 1 & \\
\hline 251 & 66-DY-162 & 3 & 661620 & 2 & \\
\hline 252 & 66-DY-163 & 3 & 661630 & 2 & \\
\hline 253 & 66-DY-164 & 3 & 661640 & 2 & \\
\hline 254 & 66-DY-165 & 3 & 661650 & 2 & \\
\hline 255 & 67-HO-165 & 3 & 671650 & 3 & $671650 / 1.000 / 1$ \\
\hline
\end{tabular}


Appendix B. Depletion chain
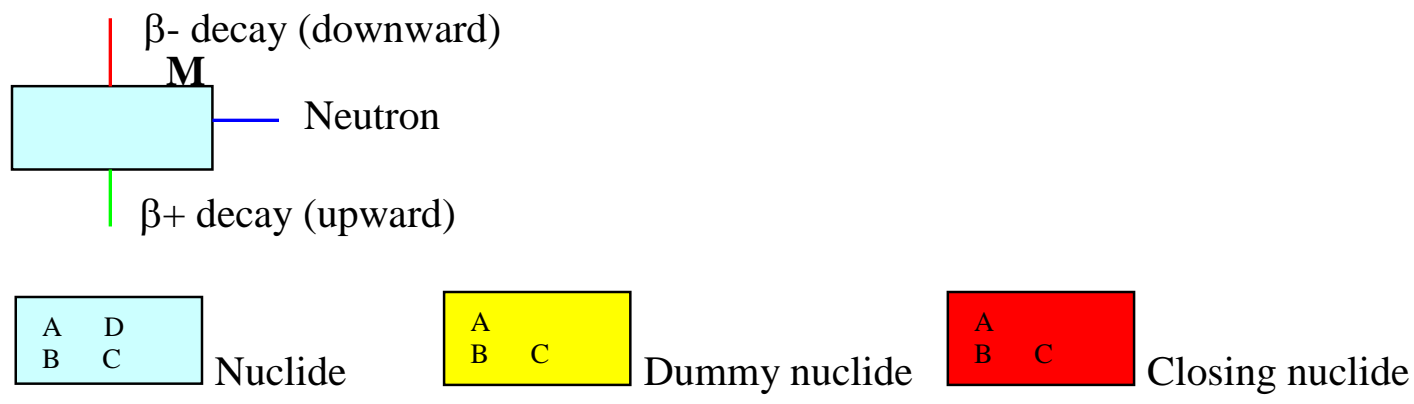
A : Nuclide
B : Nuclide Id. in library
C : Decay half life (s)
D : Decay type

$\mathrm{M}$ : Both stable and meta-stable

\section{[Depletion chain of fission product nuclides]}

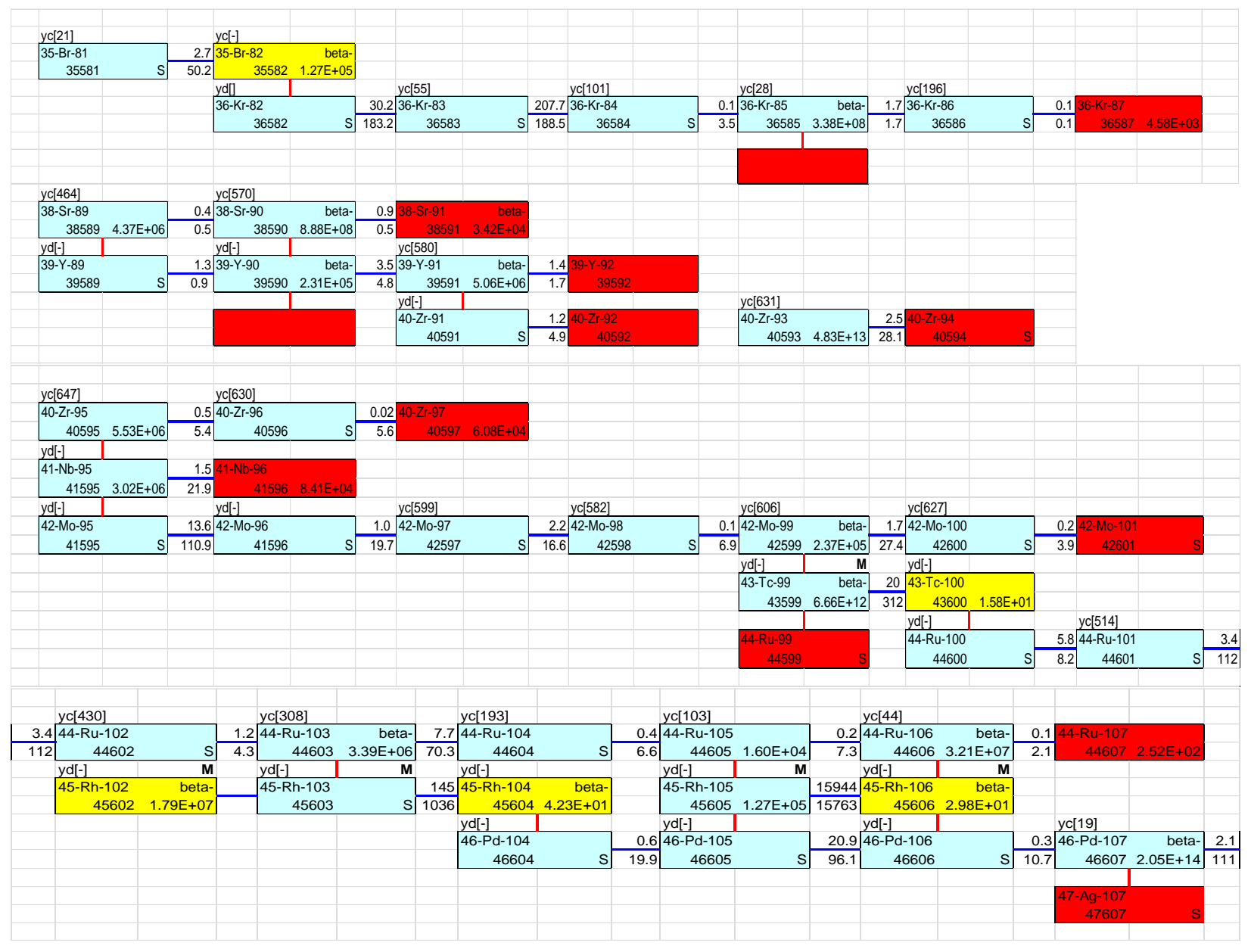



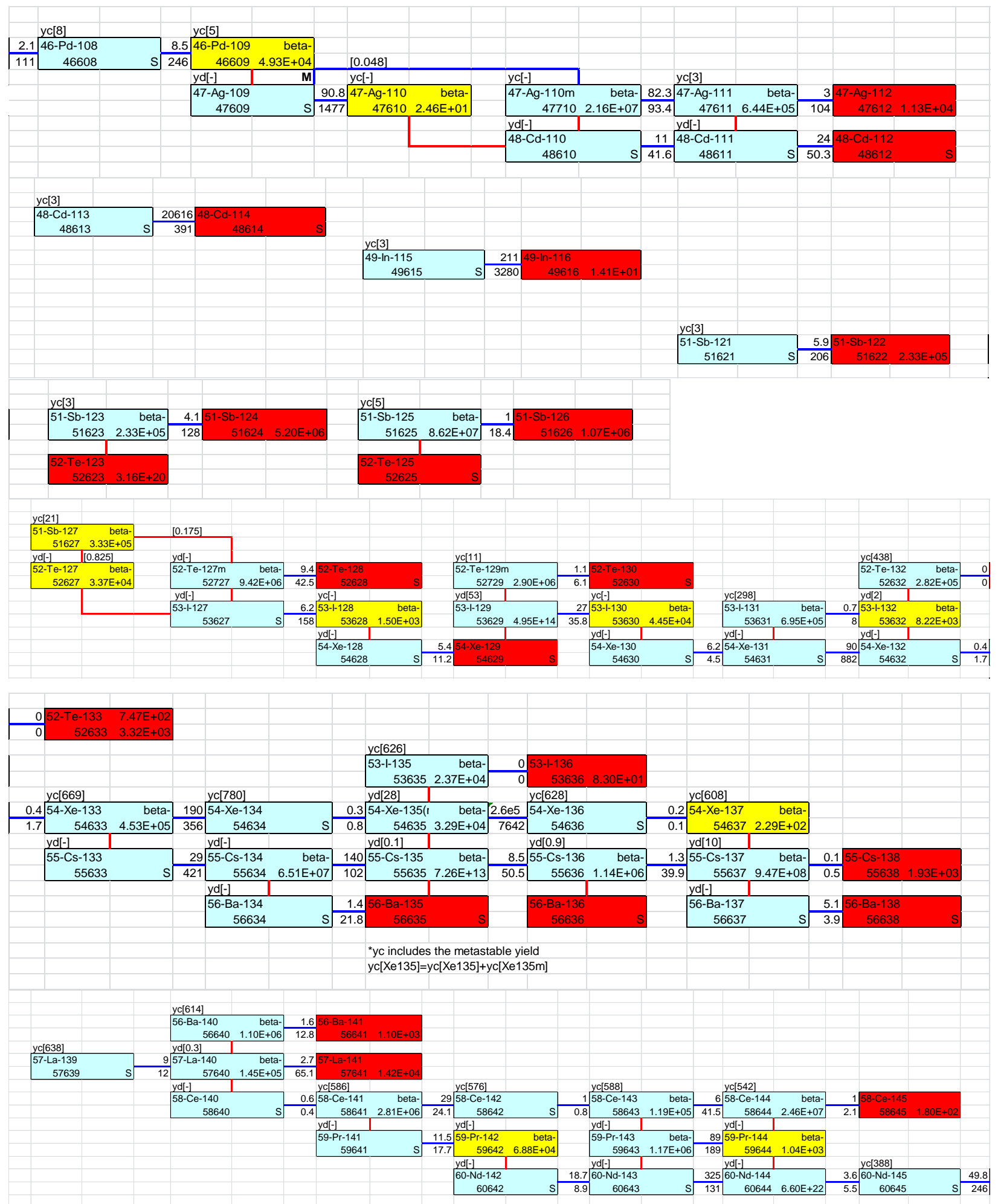


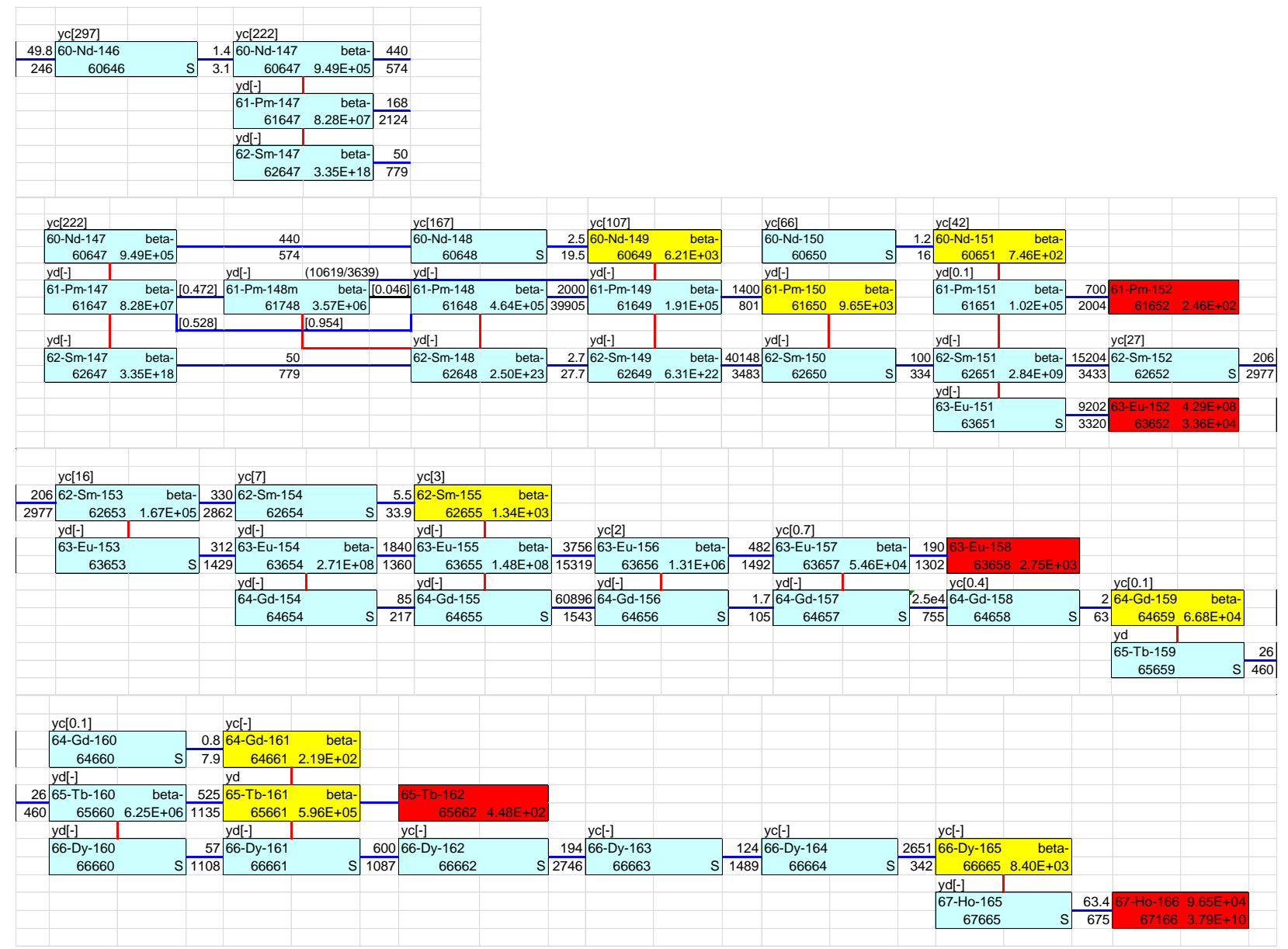




\section{[Depletion chain of heavy metal nuclides]}
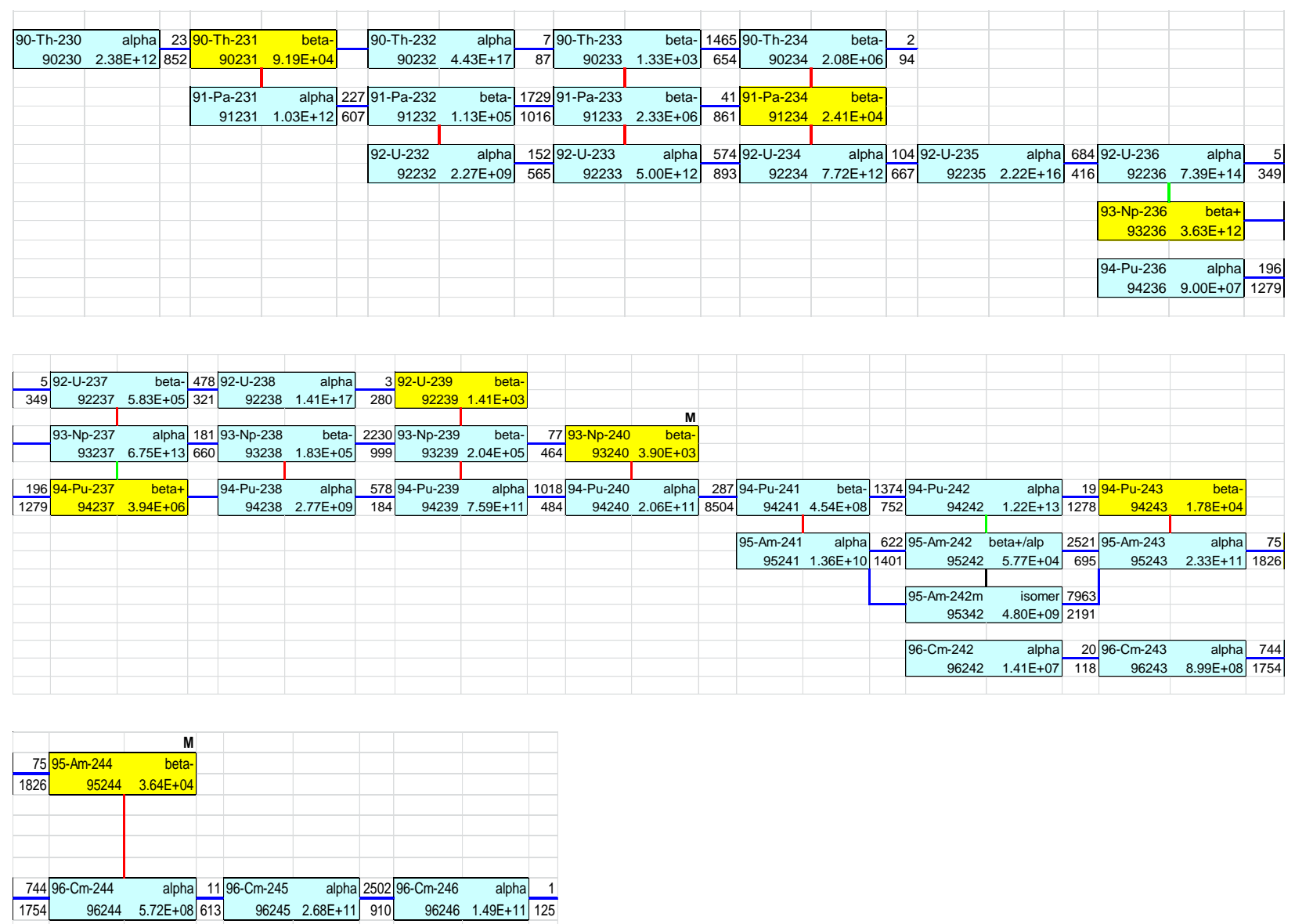


\section{[Depletion chain of activation nuclides]}

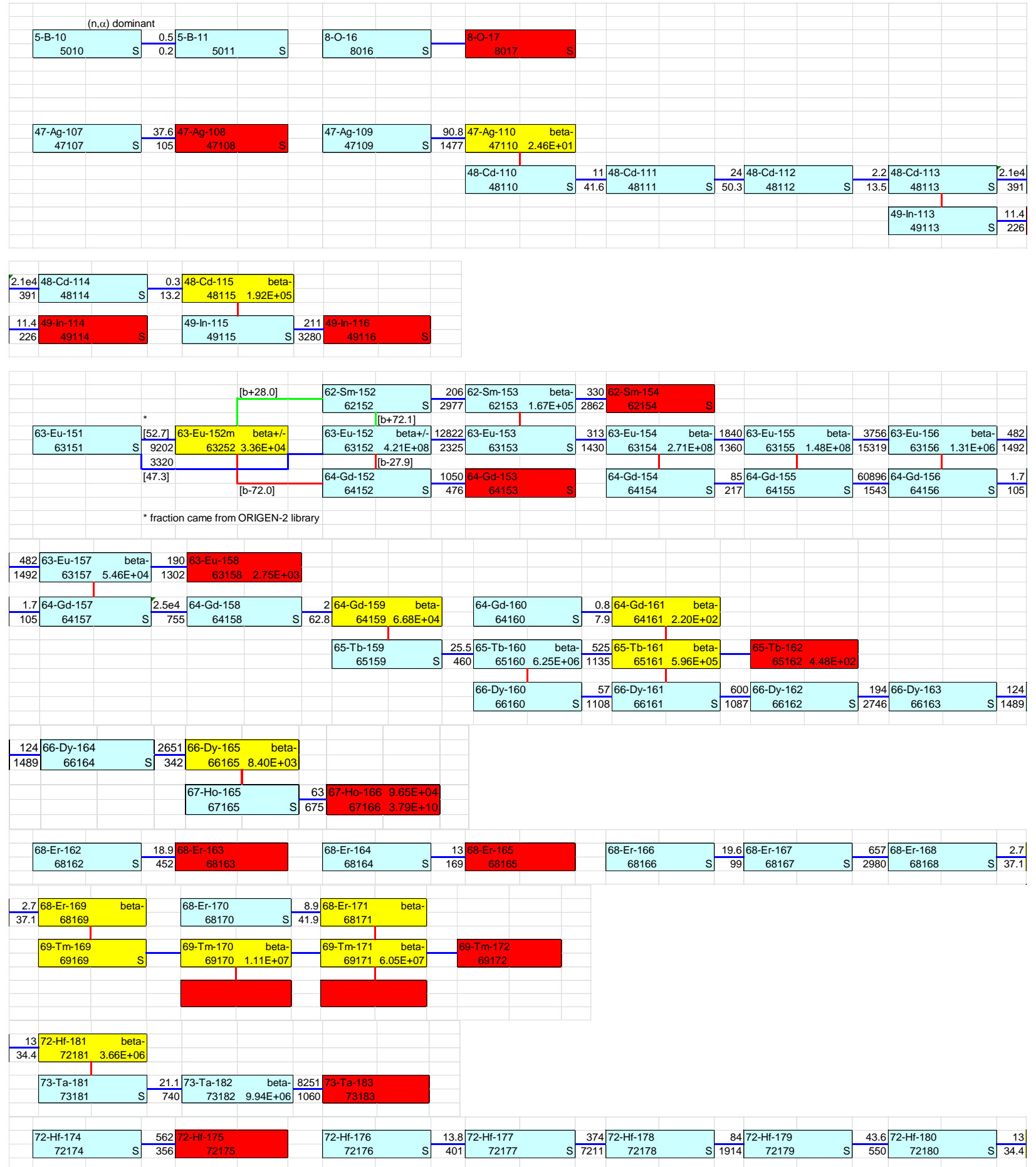

